\title{
Amine-Promoted, Organocatalytic Aziridination of Enones
}

\author{
Alan Armstrong, Carl A. Baxter, Scott G. Lamont, Andrew R. Pape and Richard Wincewicz
}

Department of Chemistry, Imperial College London, South Kensington, London SW7 2AZ, UK

E-mail: A.Armstrong@imperial.ac.uk

\section{Supporting Information}

\section{Contents}

\begin{tabular}{|c|c|}
\hline General Information & Page S1 \\
\hline Preparation of reagents and substrates & Page $\mathrm{S} 1$ \\
\hline $\begin{array}{l}\text { In situ hydrazinium salt formation and aziridination of } \\
\text { chalcones }\end{array}$ & Page S3 \\
\hline $\begin{array}{l}\text { Investigation of amine and solvent on the aziridination } \\
\text { process }\end{array}$ & Page S4 \\
\hline Scope of the aziridination procedure & Page S5 \\
\hline $\begin{array}{l}\text { Sub-stoichiometric amine in the aziridination of } \\
\text { chalcones }\end{array}$ & Page S8 \\
\hline $\begin{array}{l}\text { In situ hydrazinium salt formation and enantioselective } \\
\text { aziridination of chalcone }\end{array}$ & Page S8 \\
\hline $\begin{array}{l}\text { Investigation into the enantioselective aziridination of } \\
\text { chalcone using quinine }\end{array}$ & Page S9 \\
\hline${ }^{1} \mathrm{H}$ and ${ }^{13} \mathrm{C}$ NMR spectra & Page S10 \\
\hline
\end{tabular}

General: All reactions were conducted in oven $-\left(160^{\circ} \mathrm{C}\right)$ or flame-dried glassware under an inert atmosphere of dry $\mathrm{N}_{2}$ unless otherwise stated. Melting points were obtained using a Reichert hot plate microscope and are uncorrected. Infrared (IR) spectra were recorded on a Mattson Satellite FTIR spectrometer, $v_{\max }$ in $\mathrm{cm}^{-1}$. ${ }^{1} \mathrm{H}$ NMR spectra were recorded on a Bruker AV 400, DRX 400 or AV 500 (400 or $500 \mathrm{MHz}$ ) spectrometer. Chemical shifts are reported in ppm with the solvent resonance resulting from incomplete deuteration as the internal standard $\left(\mathrm{CDCl}_{3}: 7.26\right)$. Data are reported as follows: chemical shift, multiplicity ( $\mathrm{s}=$ singlet, $\mathrm{d}=$ doublet, $\mathrm{t}=$ triplet, $\mathrm{q}=$ quartet, $\mathrm{br}=$ broad, $\mathrm{m}=$ multiplet or combinations thereof), integration, coupling constants, and assignment. ${ }^{13} \mathrm{C}$ NMR spectra were recorded on a Bruker AV 400, DRX 400 or AV 500 (100 or $125 \mathrm{MHz}$ ) spectrometer with complete proton decoupling. Chemical shifts are reported in ppm with the solvent resonance as the internal standard $\left({ }^{13} \mathrm{CDCl}_{3}:\right.$ 77.16). Enantiomer ratios were determined by chiral HPLC analysis: Chiral Technologies Chiralpak AD-H column $(4.6 \mathrm{~mm} \times 250 \mathrm{~mm})$. High resolution mass spectrometry was performed on a Micromass AutoSpec Premier. Optical rotation values were recorded on an Optical Activity Autopolarimeter.

Reagents: Solvents were purified and dried using standard methods as required: THF and diethyl ether by distillation from sodium benzophenone ketyl, dichloromethane by distillation from calcium hydride. DMF was dried by stirring with $\mathrm{MgSO}_{4}$ for $3 \mathrm{~h}$, filtered, stirred with 4 $\AA$ MS for $12 \mathrm{~h}$, and then distilled under reduced pressure. All other solvents were used as supplied unless otherwise stated. Silica gel chromatography was performed with silica gel, particle size 40-63 nm obtained from VWR International Ltd. 
All reagents were purchased from Aldrich Chemical Company, Fisher Chemicals, or Lancaster Synthesis and purified when appropriate prior to use. ${ }^{1}$ All amines used in this study were purified prior to use using standard techniques. ${ }^{1}$ All chalcone derivatives in this study were purified by recrystallisation from hot ethanol prior to use.

Acetophenone: Aldrich

tert-Butyl acrylate: Aldrich

Chalcone: Lancaster (recrystallised from hot ethanol prior to use)

Dioxane: Aldrich

Diphenylphosphinyl chloride: Acros

3-Furaldehyde: Aldrich

Hydroxylamine hydrochloride: Lancaster

Substrates: The aromatic chalcone substrates were either bought or could be made utilising a base catalysed Claisen-Schmidt condensation reaction between the acetophenone derivative and corresponding aldehyde (see below). ${ }^{2}$ The alkyl chalcone derivatives were prepared using a Wittig reaction between phenacyl triphenylphosphonium bromide and the corresponding aldehyde (see below) according to the procedure of Pedersen. ${ }^{3}$

\section{Representative example for the preparation of chalcone $6:^{2}$}

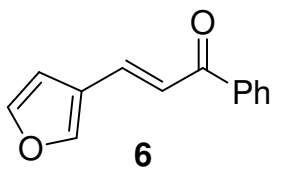

Acetophenone $(2.79 \mathrm{~mL}, 23.9 \mathrm{mmol})$ was added to a mixture of $\mathrm{MeOH}$ $(57.5 \mathrm{~mL})$ and $\mathrm{H}_{2} \mathrm{O}(11.5 \mathrm{~mL})$, and finely ground $\mathrm{KOH}(2.69 \mathrm{~g}, 47.9$ mmol) was added. The solution was allowed to stir at $\mathrm{rt}$ for $10 \mathrm{~min}$, then 3 -furaldehyde $(2.00 \mathrm{~mL}, 23.9 \mathrm{mmol})$ was added dropwise over $5 \mathrm{~min}$. The dark brown solution was allowed to stir at $\mathrm{rt}$ for $2 \mathrm{~h}$, after which tlc analysis showed the reaction was complete (as the reaction progresses a fine precipitate formed). Water $(80 \mathrm{~mL})$ and saturated $\mathrm{NH}_{4} \mathrm{Cl}$ solution $(50 \mathrm{~mL}$ ) were added and the mixture was extracted with ether (3 $\times 100 \mathrm{~mL})$. The ether was washed with brine $(50 \mathrm{~mL})$, dried $\left(\mathrm{Na}_{2} \mathrm{SO}_{4}\right)$ and concentrated in vacuo. The crude chalcone was purified by recrystallisation from hot ethanol to give the chalcone 6 (2.38 g, $12.0 \mathrm{mmol}, 50 \%)$ as a light brown solid. ${ }^{1} \mathrm{H} \mathrm{NMR}\left(400 \mathrm{MHz}, \mathrm{CDCl}_{3}\right)$ : $\delta$ 8.01-7.98 (m, 2H, PhH), 7.22 (d, 2H, J = 15.4 Hz, CH=CH, ArH), 7.60-7.56 (m, 1H, PhH), 7.51-7.48 (m, 3H, PhH, ArH), $7.25(\mathrm{~d}, 1 \mathrm{H}, J=15.4 \mathrm{~Hz}, \mathrm{CH}=\mathrm{CH}), 6.71$ (s, 1H, ArH). ${ }^{13} \mathrm{C}$ NMR $\left(100 \mathrm{MHz}, \mathrm{CDCl}_{3}\right) \delta 190.5,145.6,144.6,138.2,134.9,132.8,128.7,128.5,123.3$, 122.1, 107.5.

\section{Representative example for the preparation of $(E)$-1-phenylhept-2-en-1-one $7:^{3}$}

O Sodium hydroxide $(2.0 \mathrm{M}, 2.5 \mathrm{~mL})$ was added to a solution of phenacyl triphenylphosphonium bromide $(1.00 \mathrm{~g}, 2.17 \mathrm{mmol})$ in $\mathrm{CH}_{2} \mathrm{Cl}_{2}(5 \mathrm{~mL})$ and the resulting mixture allowed to stir at $\mathrm{rt}$ for $14 \mathrm{~h}$. The two phases were separated and the aqueous layer was extracted with $\mathrm{CH}_{2} \mathrm{Cl}_{2}(10 \mathrm{~mL})$. The $\mathrm{CH}_{2} \mathrm{Cl}_{2}$ extracts were combined, and to this solution was added valeraldehyde $(1.39 \mathrm{~mL}, 13.0 \mathrm{mmol})$ dropwise over $1 \mathrm{~h}$. The solution was allowed to stir at $\mathrm{rt}$ for $1 \mathrm{~h}$, then concentrated in vacuo. The crude chalcone derivative was purified by column chromatography (100\% petroleum ether to $10 \%$ EtOAc in petroleum ether), which gave $(E)$ 1-phenylhept-2-en-1-one 7 (229 mg, $1.22 \mathrm{mmol}, 56 \%)$ as a clear oil. ${ }^{1} \mathrm{H}$ NMR (400 MHz,

\footnotetext{
${ }^{1}$ Purification of Laboratory Chemicals; W. L. F. Armarego, D. D. Perrin, 1998.

${ }^{2}$ A slight modification of the reported procedure was performed, see: a) B. A. Bhat, K. L. Dhar, S. C. Puri, A. K. Saxena, M. Shanmugavel, G. N. Qazi, Bio. Med. Chem. Lett. 2005, 15, 3177-3180; b) E. P. Kohler, H. M. Chadwell, Org. Synth. 1922, 2, 1-2; 1942, Coll. Vol. 1, 78-79.

${ }^{3}$ F. D. Therkelson, A.-L. L. Hansen, E. B. Pedersen, C. Nielsen, Org. Biomol. Chem. 2003, 1, 2908-2918.
} 
$\left.\mathrm{CDCl}_{3}\right): \delta$ 7.93-7.91 (m, 2H, PhH), 7.56-7.53 (m, 1H, PhH), 7.48-7.44 (m, 2H, PhH), 7.07 (dt, $1 \mathrm{H}, J=15.3,6.9 \mathrm{~Hz}, \mathrm{CH}_{2} \mathrm{CH}=\mathrm{CH}$ ), 6.87 (dd, $1 \mathrm{H}, J=15.3,1.3 \mathrm{~Hz}, \mathrm{CH}_{2} \mathrm{CH}=\mathrm{CH}$ ), 2.33 (tdd, $\left.2 \mathrm{H}, J=7.3,6.9,1.3 \mathrm{~Hz}, \mathrm{CH}_{2} \mathrm{CH}=\mathrm{CH}\right), 1.51$ (tt, $2 \mathrm{H}, J=7.3,7.3 \mathrm{~Hz}, \mathrm{CH}_{3} \mathrm{CH}_{2} \mathrm{CH}_{2}$ ), 1.38 (apparent sx, 2H, $\left.J=7.3 \mathrm{~Hz}, \mathrm{CH}_{3} \mathrm{CH}_{2} \mathrm{CH}_{2}\right), 0.93\left(\mathrm{t}, 3 \mathrm{H}, J=7.3 \mathrm{~Hz}, \mathrm{CH}_{3}\right) .{ }^{13} \mathrm{C} \mathrm{NMR}(100$ $\left.\mathrm{MHz}, \mathrm{CDCl}_{3}\right) \delta 191.1,150.2,138.1,132.6,128.6,125.9,32.6,30.3,22.4,13.9$.

\section{Preparation of $O$-diphenylphosphinyl hydroxylamine 4, $\mathrm{DppONH}_{2}$}

There are numerous methods for the preparation of $\mathrm{DppONH}_{2}$, however we have found the most useful and straightforward to be a method reported by Colvin et al in 1982, ${ }^{4}$ which has since been used successfully in $1998 .^{5}$<smiles>NOP(=O)(P)P</smiles>

Aqueous $\mathrm{NaOH}\left(1.94 \mathrm{~g}, 48.6 \mathrm{mmol}\right.$, in $\left.7.00 \mathrm{~mL} \mathrm{H}_{2} \mathrm{O}, 7.1 \mathrm{M}\right)$ was added to a stirred solution of hydroxylamine hydrochloride $\left(3.96 \mathrm{~g}, 57.0 \mathrm{mmol}\right.$ ) in $\mathrm{H}_{2} \mathrm{O}$ $(8.5 \mathrm{~mL})$. Dioxane $(30 \mathrm{~mL})$ was added and the solution cooled using an ice/salt bath. Diphenylphosphinyl chloride $(5.00 \mathrm{~g}, 21.1 \mathrm{~mL})$ in dioxane $(25$ $\mathrm{mL}$ ) was added in one portion with vigorous stirring. Stirring continued for 4 min as copious precipitation ensued. Water $(30 \mathrm{~mL})$ was added and the slurry was filtered, washing with cold water $(30 \mathrm{~mL})$. The white tacky solid was dried under vacuum in a desicator $(3 \mathrm{~h})$, then purified by stirring as a slurry with aqueous $\mathrm{NaOH}(50 \mathrm{~mL}, 0.25 \mathrm{M})$ at 0 ${ }^{\circ} \mathrm{C}$ for $30 \mathrm{~min}$. The product was filtered, washing with cold water $(30 \mathrm{~mL})$, then dried under vacuum in a desicator to give $O$-diphenylphosphinyl hydroxylamine 4 (3.00 g, $12.9 \mathrm{mmol}$, $61 \%$ ) as a white powdery solid: m.p. $>135{ }^{\circ} \mathrm{C}$, gradual decomp. (lit. $>130{ }^{\circ} \mathrm{C}$, gradual decomp. $\left.{ }^{6}\right) . \quad{ }^{1} \mathrm{H}$ NMR $\left(400 \mathrm{MHz}, \mathrm{CDCl}_{3}\right): \delta$ 7.87-7.82 (m, 4H, PhH), 7.57-7.54 (m, 2H, $\mathrm{PhH}$ ), 7.50-7.47 (m, 4H, PhH), 5.9 (br s, 2H, $\left.\left.\mathrm{NH}_{2}\right) .{ }^{31} \mathrm{P} \mathrm{NMR} \mathrm{(162} \mathrm{MHz,} \mathrm{CDCl}_{3}\right) \delta$ 37.6. $m / z, 234\left(\mathrm{M}^{+}+\mathrm{H}, 100 \%\right), 201\left(\mathrm{M}^{+}-\mathrm{ONH}_{2}, 25 \%\right)$.

\section{In situ hydrazinium salt formation and aziridination of chalcones}

A note regarding the general procedure: The representative procedure below is for $\mathrm{N}$ methylmorpholine (NMM) as amine and $\mathrm{MeCN}$ used as solvent. The same procedure applies for N-methylpyrrolidine (NMP) as amine and other solvents.

\section{General procedure for the aziridination using $\mathrm{DppONH}_{2}$}

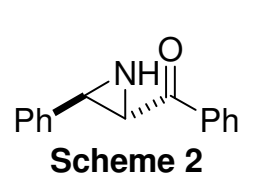

NMM $(28 \mu \mathrm{L}, 0.25 \mathrm{mmol})$ was added dropwise over 1 min to a solution of $\mathrm{DppONH}_{2}(59 \mathrm{mg}, 0.25 \mathrm{mmol})$ in $\mathrm{MeCN}(4 \mathrm{~mL})$ at $\mathrm{rt}$ under $\mathrm{N}_{2}$. The white mixture was allowed to stir for $30 \mathrm{~min}$, then $\mathrm{NaOH}(19 \mathrm{mg}, 0.48 \mathrm{mmol})$ and chalcone $(50 \mathrm{mg}, 0.24 \mathrm{mmol})$ were added sequentially. The mixture was allowed to stir at $\mathrm{rt}$ for $10 \mathrm{~h}$ (tlc indicated reaction was complete), then quenched by the addition of saturated $\mathrm{NH}_{4} \mathrm{Cl}$ solution $(2 \mathrm{~mL})$. The solution was extracted with $\mathrm{CH}_{2} \mathrm{Cl}_{2}(3 \times 10 \mathrm{~mL})$, the organics combined, dried $\left(\mathrm{Na}_{2} \mathrm{SO}_{4}\right)$ and concentrated in vacuo. Purification by column chromatography $(100 \%$ petroleum ether to $10 \%$ EtOAc in petroleum ether) gave the aziridine $(47.2 \mathrm{mg}, 0.211 \mathrm{mmol}, 88 \%)$ as a white solid: m.p. ${ }^{99-101}{ }^{\circ} \mathrm{C}$ (lit. $\left.100-101{ }^{\circ} \mathrm{C}^{7}\right)$. Data matches previously reported values. ${ }^{1} \mathrm{H} \mathrm{NMR}\left(400 \mathrm{MHz}, \mathrm{CDCl}_{3}\right): \delta 8.00$ $(\mathrm{d}, 1 \mathrm{H}, J=8.8 \mathrm{~Hz}, \mathrm{PhH}), 8.00(\mathrm{~d}, 1 \mathrm{H}, J=8.8 \mathrm{~Hz}, \mathrm{PhH}), 7.61(\mathrm{td}, 1 \mathrm{H}, J=7.4,1.2 \mathrm{~Hz}, \mathrm{PhH})$, 7.49 (t, 2H, $J=7.4 \mathrm{~Hz}, \mathrm{PhH}), 7.39-7.30(\mathrm{~m}, 5 \mathrm{H}, \mathrm{PhH}), 3.52(\mathrm{dd}, 1 \mathrm{H}, J=7.9,2.1 \mathrm{~Hz}$, CHNH), $3.18(\mathrm{dd}, 1 \mathrm{H}, J=9.1,2.1 \mathrm{~Hz}, \mathrm{CHNH}), 2.68$ (br dd, $1 \mathrm{H}, J=9.1,7.9 \mathrm{~Hz}, \mathrm{NH}) .{ }^{13} \mathrm{C}$

\footnotetext{
${ }^{4}$ E. W. Colvin, G. W. Kirby, A. C. Wilson, Tetrahedron Lett. 1982, 23, 3835-3836.

${ }^{5}$ M. G. Stocksdale, S. Ramurthy, M. J. Miller, J. Org. Chem. 1998, 63, 1221-1225.

${ }^{6}$ M. J. P. Harger, J. Chem. Soc., Perkin Trans. 1, 1981, 3284-3288.

${ }^{7}$ J. Xu, P. Jiao, J. Chem. Soc., Perkin Trans. 1 2002, 1491-1493.
} 
NMR $\left(100 \mathrm{MHz}, \mathrm{CDCl}_{3}\right) \delta 195.8,138.4,136.0,133.9,128.9,128.6,128.4,128.0,126.3$, 44.2, 43.6.

\section{Investigation of amine and solvent on the aziridination process}

After the initial success of performing in situ amination of NMM and subsequent aziridination of chalcone, we set out to investigate the effect of amine and solvent on the reaction. A range of amines (aromatic, secondary and tertiary) were studied in the reaction and the results are shown in Table 4. Entry 1 shows the original result with NMM; this promoter is effective in several solvents, with $\mathrm{CH}_{2} \mathrm{Cl}_{2}$ and DMF giving comparable conversion to $\mathrm{MeCN}$ (entries 2 and 5). Pyridines and secondary amines are not effective amines for this reaction, with DMAP, pyridine, morpholine and diisopropylamine giving no aziridine after 24 hours (entries 18-24). With other tertiary amines, DABCO was found to be inferior to NMM under our in situ reaction conditions (entry 6). Quinuclidine gave poor results in $\mathrm{MeCN}$, but reasonable conversion in $\mathrm{CH}_{2} \mathrm{Cl}_{2}$ and DMF (entries 7-9). N-Methylpiperidine was a poor promoter in MeCN (entry 10); N-methylpyrrolidine (NMP) gave equally good results as NMM (entries 11, 12 and 15) while triethylamine worked surprisingly well (entries 16 and 17). Overall, these results indicated NMM and NMP as the best promoters. We do not currently have a convincing rationale for the high solvent dependency on rates, but it may be associated with the relative solubilities of the intermediate hydrazinium salts.

Table 4. Investigation of the effect of amine and solvent on the aziridination method ${ }^{a}$

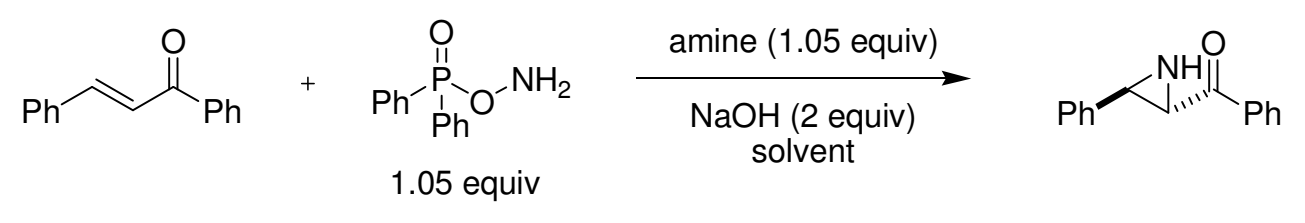

\begin{tabular}{|c|c|c|c|c|}
\hline Entry & Amine & $\begin{array}{l}\text { Solvent; } \\
\text { Time (h) }\end{array}$ & Conv $(\%)^{b}$ & Yield $(\%)^{c}$ \\
\hline 1 & NMM & $\mathrm{MeCN} ; 9$ & $>95$ & 88 \\
\hline 2 & NMM & $\mathrm{CH}_{2} \mathrm{Cl}_{2} ; 3$ & 90 & 88 \\
\hline 3 & NMM & THF; 5 & - & 86 \\
\hline 4 & NMM & Toluene; 24 & $\sim 42$ & - \\
\hline 5 & NMM & DMF; 6 & 88 & 82 \\
\hline 6 & DABCO & $\mathrm{MeCN} ; 24$ & 65 & - \\
\hline 7 & Quinuclidine & $\mathrm{MeCN} ; 24$ & $<2$ & - \\
\hline 8 & Quinuclidine & $\mathrm{CH}_{2} \mathrm{Cl}_{2} ; 10$ & 89 & 80 \\
\hline 9 & Quinuclidine & DMF; 24 & 70 & 65 \\
\hline 10 & $\mathrm{~N}$-methylpiperidine & $\mathrm{MeCN} ; 24$ & $<2$ & - \\
\hline 11 & NMP & $\mathrm{MeCN} ; 8$ & $>98$ & 75 \\
\hline 12 & NMP & $\mathrm{CH}_{2} \mathrm{Cl}_{2} ; 2$ & $>95$ & 94 \\
\hline 13 & NMP & THF; 10 & 62 & 58 \\
\hline 14 & NMP & Toluene; 24 & $>98$ & 89 \\
\hline 15 & NMP & DMF; 2 & $>98$ & 88 \\
\hline 16 & Triethylamine & $\mathrm{MeCN} ; 24$ & 80 & 57 \\
\hline 17 & Triethylamine & $\mathrm{CH}_{2} \mathrm{Cl}_{2} ; 24$ & 70 & 50 \\
\hline 18 & DMAP & $\mathrm{MeCN} ; 24$ & $<2$ & - \\
\hline 19 & Pyridine & $\mathrm{MeCN} ; 24$ & $<2$ & - \\
\hline 20 & Pyridine & $\mathrm{CH}_{2} \mathrm{Cl}_{2} ; 24$ & $\sim 14^{d}$ & - \\
\hline 21 & Morpholine & $\mathrm{MeCN} ; 24$ & $<2$ & - \\
\hline 22 & Diisopropylamine & $\mathrm{MeCN} ; 24$ & $<2$ & - \\
\hline 23 & Morpholine & $\mathrm{CH}_{2} \mathrm{Cl}_{2} ; 24$ & $<2$ & - \\
\hline 24 & Diisopropylamine & $\mathrm{CH}_{2} \mathrm{Cl}_{2} ; 24$ & $<2$ & - \\
\hline
\end{tabular}

${ }^{a}$ Reactions performed on $0.12 \mathrm{mmol}$ scale. ${ }^{b}$ Based on ${ }^{1} \mathrm{H}$ NMR analysis of the crude reaction mixture. ${ }^{c}$ Isolated yields after column chromatography. ${ }^{d}$ Product was identified in the crude ${ }^{1} \mathrm{H}$ NMR as reduction of the alkene, presumably by diimide formation. 


\section{Scope of the aziridination procedure}

\section{(3-(3-Nitrophenyl)aziridin-2-yl)(phenyl)methanone}

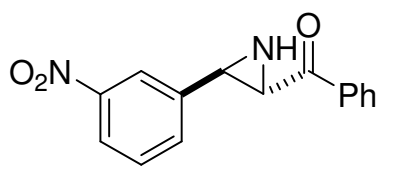

Table 1, entry 1

The general procedure for aziridination using $\mathrm{DppONH}_{2}, \mathrm{NMM}$ and $\mathrm{MeCN}$ was followed. The reaction turned a deep purple colour and gave the aziridine ( $49 \mathrm{mg}, 0.18 \mathrm{mmol}, 75 \%$ ) as a white solid: m.p. $120-122{ }^{\circ} \mathrm{C}$. IR (neat) $v 1661,1527,1351,1259 \mathrm{~cm}^{-1} .{ }^{1} \mathrm{H}$ NMR (400 MHz, $\left.\mathrm{CDCl}_{3}\right): \delta 8.24(\mathrm{~s}, 1 \mathrm{H}, \mathrm{PhH}), 8.17(\mathrm{~d}, 1 \mathrm{H}, J=8.2$ $\mathrm{Hz}, \mathrm{PhH}), 8.00$ (d, 2H, J= 7.3 Hz, PhH), $7.72(\mathrm{~d}, 1 \mathrm{H}, J=7.7 \mathrm{~Hz}, \mathrm{PhH}), 7.64$ (t, 1H, J = 7.4 $\mathrm{Hz}, \mathrm{PhH}), 7.56-7.49$ (m, 3H, PhH), 3.53 (dd, 1H, $J=7.7,2.0 \mathrm{~Hz}, \mathrm{CHNH}), 3.27$ (dd, 1H, $J=$ 8.9, $2.0 \mathrm{~Hz}, \mathrm{CHNH}), 2.79-2.74$ (br m, 1H, NH). ${ }^{13} \mathrm{C} \mathrm{NMR}\left(100 \mathrm{MHz}, \mathrm{CDCl}_{3}\right) \delta 194.9$, 148.5, 140.7, 135.6, 134.2, 132.4, 129.5, 128.9, 128.4, 122.8, 121.3, 43.9, 42.1. HRMS (CI, $\left.\mathrm{NH}_{3}\right): m / z\left[\mathrm{M}^{+}+\mathrm{H}\right]$ calcd for $\mathrm{C}_{15} \mathrm{H}_{13} \mathrm{~N}_{2} \mathrm{O}_{3}: 269.0926$; found: 269.0932 .

\section{4-(3-Benzoylaziridin-2-yl)benzonitrile}

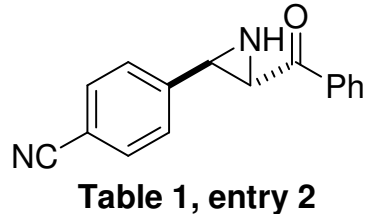

The general procedure for aziridination using $\mathrm{DppONH}_{2}, \mathrm{NMM}$ and $\mathrm{MeCN}$ was followed. The reaction turned an orange/red colour and gave the aziridine (33 $\mathrm{mg}, 0.13 \mathrm{mmol}, 62 \%$ ) as a slight yellow solid: m.p. $74-75{ }^{\circ} \mathrm{C}$ (lit. $113.5-113.7{ }^{\circ} \mathrm{C}^{8}$ ). Data matches previously reported values: ${ }^{1} \mathrm{H}$ NMR $\left(400 \mathrm{MHz}, \mathrm{CDCl}_{3}\right): \delta 7.98(\mathrm{~d}, 2 \mathrm{H}, J=7.3$ $\mathrm{Hz}, \mathrm{PhH}), 7.67-7.61$ (m, 3H, PhH), 7.53-7.47 (m, 4H, PhH), 3.49

(br d, 1H, $J=5.7 \mathrm{~Hz}, \mathrm{CHNH}$ ), 3.20 (br d, $1 \mathrm{H}, J=7.2 \mathrm{~Hz}, \mathrm{CHNH}$ ), 2.79-2.70 (br m, 1H, $\mathrm{NH}) .{ }^{13} \mathrm{C} \mathrm{NMR}\left(100 \mathrm{MHz}, \mathrm{CDCl}_{3}\right) \delta 194.9,143.8,135.5,134.2,132.4,128.9,128.3,127.0$, $118.7,111.5,44.1,42.5$.

\section{Phenyl(3-p-tolylaziridin-2-yl)methanone}

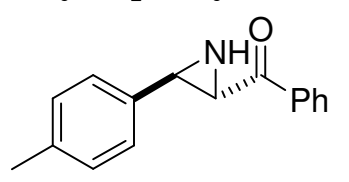

Table 1, entry 3

The general procedure for aziridination using $\mathrm{DppONH}_{2}, \mathrm{NMM}$ and $\mathrm{MeCN}$ was followed. The reaction turned a slight yellow colour and gave the aziridine (44 $\mathrm{mg}, 0.185 \mathrm{mmol}, 78 \%$ ) as a white solid: m.p. 107-108 ${ }^{\circ} \mathrm{C}$ (lit. $106-107{ }^{\circ} \mathrm{C}^{7}$ ). Data matches previously reported values: ${ }^{1} \mathrm{H}$ NMR $\left(400 \mathrm{MHz}, \mathrm{CDCl}_{3}\right): \delta 8.03-8.00(\mathrm{~m}, 2 \mathrm{H}, \mathrm{PhH})$, 7.65-7.62 (m, 1H, PhH), 7.53-7.50 (m, 2H, PhH), 7.30-7.28 (m,

2H, $\mathrm{PhH}$ ), 7.21-7.19 (m, 2H, PhH), 3.52 (br s, 1H, CHNH), 3.18 (br s, 1H, CHNH), 2.68 (br, 1H, NH), $2.39\left(\mathrm{~s}, 3 \mathrm{H}, \mathrm{CH}_{3}\right) .{ }^{13} \mathrm{C} \mathrm{NMR}\left(100 \mathrm{MHz}, \mathrm{CDCl}_{3}\right) \delta$ 195.8, 137.7, 136.0, 135.4, $133.8,129.3,128.8,128.3,126.1,119.0,44.2,43.6,21.2$.

\section{(3-(4-Methoxyphenyl)aziridin-2-yl)(phenyl)methanone}<smiles>COc1ccc(C2NC2C(=O)c2ccccc2)cc1</smiles>

Table 1, entry 4

The general procedure for aziridination using $\mathrm{DppONH}_{2}, \mathrm{NMP}$ and $\mathrm{CH}_{2} \mathrm{Cl}_{2}$ was followed. The reaction turned a yellow colour and gave the aziridine (47.9 $\mathrm{mg}, 0.19 \mathrm{mmol}, 79 \%$ ) as a yellow oil. Data matches previously reported values: ${ }^{1} \mathrm{H}$ NMR $(400 \mathrm{MHz}$, $\left.\mathrm{CDCl}_{3}\right): \delta$ 8.00-7.98 (m, 2H, PhH), 7.63-7.59 (m, 1H, PhH), 7.51-7.47 (m, 2H, PhH), $7.29(\mathrm{~d}, 2 \mathrm{H}, J=8.7 \mathrm{~Hz}, \mathrm{ArH}), 6.90(\mathrm{~d}$, $2 \mathrm{H}, J=8.7 \mathrm{~Hz}, \mathrm{ArH}$ ), 3.82 (s, 3H, CH$)_{3}, 3.48-3.47$ (br m, 1H, CHNH), 3.15-3.13 (br m, 1H, $\mathrm{CHNH}$ ), 2.71-2.58 (br m, 1H, NH). ${ }^{13} \mathrm{C} \mathrm{NMR}\left(100 \mathrm{MHz}, \mathrm{CDCl}_{3}\right) \delta 195.9,159.5,136.0$, $133.9,130.5,128.9,128.4,127.4,114.1,55.4,44.3,43.4$.

\footnotetext{
${ }^{8}$ Prepared by a two-step asymmetric aziridination of chalcones using rare-earth metal catalyzed Michael addition of hydroxylamines, see: X. L. Jin, H. Sugihara, K. Daikai, H. Tateishi, Y. Z. Jin, H. Furuno, J. Inanaga, Tetrahedron 2002, 58, 8321-8329.
} 


\section{(4-Chlorophenyl)(3-phenylaziridin-2-yl)methanone}

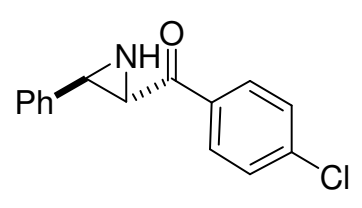

Table 1, entry 5

The general procedure for aziridination using $\mathrm{DppONH}_{2}, \mathrm{NMM}$ and $\mathrm{MeCN}$ was followed. The reaction turned a slight yellow colour and gave the aziridine $(55.5 \mathrm{mg}, 0.215 \mathrm{mmol}, 90 \%)$ as a white solid: m.p. 76-78 ${ }^{\circ} \mathrm{C}$ (lit. $76-78{ }^{\circ} \mathrm{C}^{7}$ ). Data matches previously reported values: ${ }^{1} \mathrm{H}$ NMR (400 MHz, $\left.\mathrm{CDCl}_{3}\right): \delta$ 7.95-7.92 (m, 2H, PhH), 7.48-7.45 $(\mathrm{m}, 2 \mathrm{H}, \mathrm{PhH}), 7.38-7.30(\mathrm{~m}, 5 \mathrm{H}, \mathrm{PhH}), 3.45$ (br d, $1 \mathrm{H}, J=4.8 \mathrm{~Hz}$, $\mathrm{CHNH}$ ), 3.19 (br d, 1H, $J=5.2 \mathrm{~Hz}, \mathrm{CHNH}), 2.70-2.63$ (br m, 1H, NH). ${ }^{13} \mathrm{C} \mathrm{NMR}(100$ $\left.\mathrm{MHz}, \mathrm{CDCl}_{3}\right) \delta 194.6,140.4,138.1,134.2,129.7,129.2,128.6,128.0,126.2,44.1,43.7$.

\section{(3-Phenylaziridin-2-yl)(p-tolyl)methanone}

The general procedure for aziridination using $\mathrm{DppONH}_{2}, \mathrm{NMM}$ and<smiles>Cc1ccc(C(=O)[C@@H]2NC2c2ccccc2)cc1</smiles>

Table 1, entry 6 $\mathrm{MeCN}$ was followed. The reaction turned a slight yellow colour and gave the aziridine $(48.5 \mathrm{mg}, 0.204 \mathrm{mmol}, 86 \%)$ as a slight yellow solid: m.p. 89-90 ${ }^{\circ} \mathrm{C}$ (lit. $89-90{ }^{\circ} \mathrm{C}^{7}$ ). Data matches previously reported values: ${ }^{1} \mathrm{H}$ NMR $\left(400 \mathrm{MHz}, \mathrm{CDCl}_{3}\right): \delta 7.96-7.92(\mathrm{~m}, 2 \mathrm{H}$, $\mathrm{PhH}$ ), 7.40-7.29 (m, 7H, PhH), 3.52 (br s, 1H, CHNH), 3.19 (br s, 1H, $\mathrm{CHNH}$ ), 2.69 (br, 1H, NH), 2.45 (s, 3H, $\left.\mathrm{CH}_{3}\right) .{ }^{13} \mathrm{C} \mathrm{NMR}\left(100 \mathrm{MHz}, \mathrm{CDCl}_{3}\right) \delta$ 195.2, 144.9, $138.5,133.5,129.5,129.3,128.6,128.5,127.9,126.2,44.0,43.4,21.8$.

\section{(4-Methoxyphenyl)(3-phenylaziridin-2-yl)methanone}

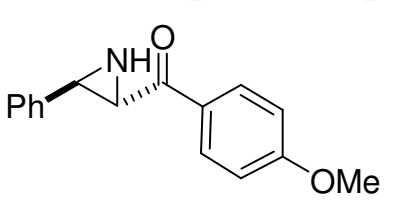

Table 1, entry 7

The general procedure for aziridination using $\mathrm{DppONH}_{2}, \mathrm{NMM}$ and $\mathrm{MeCN}$ was followed. The reaction turned a slight yellow colour and gave the aziridine $(58.5 \mathrm{mg}, 0.231 \mathrm{mmol}, 97 \%)$ as a white solid: m.p. $50-51{ }^{\circ} \mathrm{C}$ (lit. $71-72{ }^{\circ} \mathrm{C}^{7}$ ). Data matches previously reported values: ${ }^{1} \mathrm{H}$ NMR $\left(400 \mathrm{MHz}, \mathrm{CDCl}_{3}\right): \delta$ 8.00-7.97 (m, 2H, PhH), 7.37-7.29 (m, 5H, PhH), 6.97-6.94 (m, 2H, PhH), 3.90 (s, 3H, OCH $), 3.49$ (d, 1H, J = 4.4 Hz, CHNH), $3.18(\mathrm{~d}, 1 \mathrm{H}, J=5.2 \mathrm{~Hz}$, $\mathrm{CHNH}$ ), 2.70-2.64 (br m, 1H, NH). ${ }^{13} \mathrm{C}$ NMR (100 MHz, $\left.\mathrm{CDCl}_{3}\right) \delta 194.0,164.2,138.6$, $130.7,129.0,128.6,127.8,126.2,114.1,55.6,43.8,43.1$.

\section{(3-(Naphthalen-1-yl)aziridin-2-yl)(phenyl)methanone}<smiles>O=C(c1ccccc1)[C@H]1NC1c1cccc2ccccc12</smiles>

Table 1, entry 8

The general procedure for aziridination using $\mathrm{DppONH}_{2}, \mathrm{NMP}$ and $\mathrm{CH}_{2} \mathrm{Cl}_{2}$ was followed. The reaction turned an orange colour and gave the aziridine (43.9 $\mathrm{mg}, 0.16 \mathrm{mmol}, 67 \%$ ) as a slight yellow solid: m.p. $148-150{ }^{\circ} \mathrm{C}$. IR (neat) v 1664, 1450, $1261 \mathrm{~cm}^{-1}$. ${ }^{1} \mathrm{H}$ NMR $(400 \mathrm{MHz}$, $\left.\mathrm{CDCl}_{3}\right): \delta 8.07(\mathrm{~d}, 2 \mathrm{H}, J=7.4 \mathrm{~Hz}, \mathrm{ArH}), 8.01(\mathrm{~d}, 1 \mathrm{H}, J=8.3 \mathrm{~Hz}$, $\operatorname{ArH}), 7.91(\mathrm{~d}, 1 \mathrm{H}, J=8.1 \mathrm{~Hz}, \operatorname{ArH}), 7.83(\mathrm{~d}, 1 \mathrm{H}, J=8.1 \mathrm{~Hz}, \operatorname{ArH})$, $7.77(\mathrm{~d}, 1 \mathrm{H}, J=7.1 \mathrm{~Hz}, \operatorname{ArH}), 7.63(\mathrm{t}, 1 \mathrm{H}, J=7.4 \mathrm{~Hz}, \operatorname{ArH}), 7.53-7.42(\mathrm{~m}, 5 \mathrm{H}, \operatorname{ArH}), 3.78$ (br s, $1 \mathrm{H}, \mathrm{CHNH}$ ), 3.54 (br s, $1 \mathrm{H}, \mathrm{CHNH}), 2.73$ (br s, $1 \mathrm{H}, \mathrm{NH}) .{ }^{13} \mathrm{C}$ NMR $(100 \mathrm{MHz}$, $\left.\mathrm{CDCl}_{3}\right) \delta 196.3,135.9,134.0,133.5,131.9,129.0,128.9,128.5,128.2,126.5,126.1,126.0$, 125.7, 124.0, 122.8, 43.1, 41.9. HRMS $\left(\mathrm{CI}, \mathrm{NH}_{3}\right): \mathrm{m} / z\left[\mathrm{M}^{+}+\mathrm{H}\right]$ calcd for $\mathrm{C}_{19} \mathrm{H}_{16} \mathrm{NO}$ : 274.1232; found: 274.1240 . 


\section{(3-(Furan-3-yl)aziridin-2-yl)(phenyl)methanone}

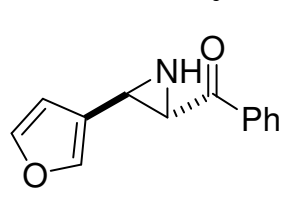

Table 1, entry 9

The general procedure for aziridination using $\mathrm{DppONH}_{2}, \mathrm{NMM}$ and $\mathrm{MeCN}$ was followed. The reaction turned a deep brown colour and gave the aziridine (46.1 mg, $0.22 \mathrm{mmol}, 90 \%)$ as a light brown solid: m.p. $76-77{ }^{\circ} \mathrm{C}$. IR (neat) v 1668, 1598, 1450, 1259, $1012 \mathrm{~cm}^{-1} .{ }^{1} \mathrm{H}$ NMR $(400$ $\left.\mathrm{MHz}, \mathrm{CDCl}_{3}\right): \delta 8.01(\mathrm{~d}, 2 \mathrm{H}, J=7.3 \mathrm{~Hz}, \mathrm{PhH}), 7.65-7.61(\mathrm{~m}, 1 \mathrm{H}, \mathrm{PhH})$, 7.53-7.49 (m, 3H, PhH, ArH), 7.41-7.40 (m, 1H, ArH), $6.42(\mathrm{~d}, 1 \mathrm{H}, J=$

$0.9 \mathrm{~Hz}, \mathrm{ArH}), 3.52$ (br s, 1H, CHNH), 3.07 (br s, $1 \mathrm{H}, \mathrm{CHNH}$ ), 2.54 (br s, $1 \mathrm{H}, \mathrm{NH}) .{ }^{13} \mathrm{C}$ NMR $\left(100 \mathrm{MHz}, \mathrm{CDCl}_{3}\right) \delta 196.0,143.6,140.7,136.0,134.0,128.9,128.4,124.1,108.5$, 42.7, 36.7. HRMS (CI, $\left.\mathrm{NH}_{3}\right): \mathrm{m} / z\left[\mathrm{M}^{+}+\mathrm{H}\right]$ calcd for $\mathrm{C}_{13} \mathrm{H}_{12} \mathrm{NO}_{2}$ : 214.0868; found: 214.0875 .

\section{(3-Butylaziridin-2-yl)(phenyl)methanone}

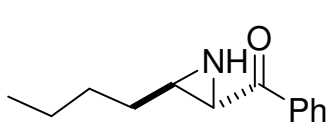

Table 1, entry 10

The general procedure for aziridination using $\mathrm{DppONH}_{2}, \mathrm{NMM}$ and $\mathrm{CH}_{2} \mathrm{Cl}_{2}$ was followed. The aziridine (40.6 $\mathrm{mg}, 0.200 \mathrm{mmol}, 83 \%$ ) was isolated as a clear oil. Data matches previously reported values: ${ }^{9}{ }^{1} \mathrm{H}$ NMR (400 MHz, $\left.\mathrm{CDCl}_{3}\right): \delta 8.04-8.01(\mathrm{~m}, 2 \mathrm{H}, \mathrm{PhH}), 7.64-7.60(\mathrm{~m}$, 1H, $\mathrm{PhH}$ ), 7.53-7.50 (m, 2H, PhH), 3.26 (s, 1H, CHNH), 2.18-2.15

(m, 1H, CHNH), 2.06 (br s, 1H, NH), 1.62-1.57 (m, 2H, CH $\left.\mathbf{H}_{2} \mathrm{CHNH}\right), 1.55-1.33$ (m, 4H, $\left.\mathrm{CH}_{3} \mathrm{CH}_{2} \mathrm{CH}_{2}\right), 0.92\left(\mathrm{t}, 3 \mathrm{H}, J=7.2 \mathrm{~Hz}, \mathrm{CH}_{3}\right),{ }^{13} \mathrm{C}$ NMR $\left(100 \mathrm{MHz}, \mathrm{CDCl}_{3}\right) \delta 197.4,136.2$, 133.7, 128.8, 128.2, 43.4, 39.9, 33.1, 29.4, 22.5, 14.0. HRMS (CI, $\left.\left.\mathrm{NH}_{3}\right): \mathrm{m} / z . \mathrm{M}^{+}+\mathrm{H}\right]$ calcd for $\mathrm{C}_{13} \mathrm{H}_{18} \mathrm{NO}$ : 204.1388; found: 204.1385.

\section{(3-Isobutylaziridin-2-yl)(phenyl)methanone}

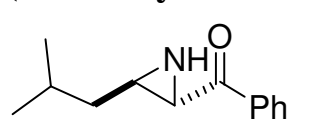

Table 1, entry 11

The general procedure for aziridination using $\mathrm{DppONH}_{2}, \mathrm{NMM}$ and $\mathrm{CH}_{2} \mathrm{Cl}_{2}$ was followed. The aziridine $(38.1 \mathrm{mg}, 0.187 \mathrm{mmol}, 78 \%$ ) was isolated as a clear oil. Data matches previously reported values: ${ }^{10}{ }^{1} \mathrm{H}$ NMR (400 MHz, $\left.\mathrm{CDCl}_{3}\right): \delta$ 8.03-8.01 (m, 2H, $\left.\mathrm{PhH}\right), 7.64-7.60(\mathrm{~m}, 1 \mathrm{H}$, PhH), 7.53-7.49 (m, 2H, PhH), 3.24 (d, 1H, $J=2.2 \mathrm{~Hz}, \mathrm{CHNH}), 2.19$ (td, $1 \mathrm{H}, J=6.2,2.2$ $\mathrm{Hz}, \mathrm{CHNH}), 2.00$ (br, 1H, NH), 1.88-1.77 (m, 1H, $\left.\mathrm{CHCH}_{2}\right), 1.55-1.45$ (m, 2H, $\left.\mathrm{CH}_{2} \mathrm{CHNH}\right)$, $0.97\left(\mathrm{~d}, 6 \mathrm{H}, J=6.6 \mathrm{~Hz}, \mathrm{CH}\left(\mathrm{CH}_{3}\right)_{2}\right) .{ }^{13} \mathrm{C} \mathrm{NMR}\left(100 \mathrm{MHz}, \mathrm{CDCl}_{3}\right) \delta 197.4,136.3,133.8$, $128.9,128.3,42.5,42.3,40.0,27.4,22.9,22.6$.

\section{(3-Phenethylaziridin-2-yl(phenyl)methanone}

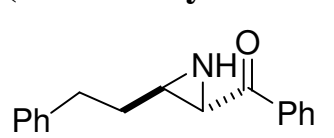

Table 1, entry 12

The general procedure for aziridination using $\mathrm{DppONH}_{2}, \mathrm{NMM}$ and $\mathrm{CH}_{2} \mathrm{Cl}_{2}$ was followed. The aziridine (37.4 $\mathrm{mg}, 0.149 \mathrm{mmol}, 62 \%$ ) was isolated as a clear oil. IR (neat) $\vee 3267,2925,2856,1668,1451,1259$ $\mathrm{cm}^{-1} . \quad{ }^{1} \mathrm{H}$ NMR $\left(400 \mathrm{MHz}, \mathrm{CDCl}_{3}\right): \delta 7.87-7.83(\mathrm{~m}, 2 \mathrm{H}, \mathrm{PhH})$, 7.63-7.60 (m, 1H, PhH), 7.50-7.46 (m, 2H, PhH), 7.28-7.19 (m, 5H,

$\mathrm{PhH}), 3.21(\mathrm{~d}, 1 \mathrm{H}, J=2.2 \mathrm{~Hz}, \mathrm{CHNH}), 2.93-2.74\left(\mathrm{~m}, 2 \mathrm{H}, \mathrm{CH}_{2}\right), 2.20(\mathrm{td}, 1 \mathrm{H}, J=6.0,2.2$ $\mathrm{Hz}, \mathrm{CHNH}), 2.03-1.87\left(\mathrm{~m}, 3 \mathrm{H}, \mathrm{CH}_{2}, \mathrm{NH}\right) .{ }^{13} \mathrm{C} \mathrm{NMR}\left(100 \mathrm{MHz}, \mathrm{CDCl}_{3}\right) \delta 197.3,141.3$, 136.1, 133.8, 128.9, 128.7, 128.5, 128.3, 126.2, 42.8, 40.0, 34.9, 33.5. HRMS (ES): $m / z ~\left[\mathrm{M}^{+}\right.$ $+\mathrm{H}]$ calcd for $\mathrm{C}_{17} \mathrm{H}_{18} \mathrm{NO}$ : 252.1388 ; found: $252.1400 ; \mathrm{m} / z\left[\mathrm{M}^{+}+\mathrm{Na}\right]$ calcd for $\mathrm{C}_{17} \mathrm{H}_{17} \mathrm{NONa}$ : 274.1208; found: 274.1216 .

\footnotetext{
${ }^{9}$ I. Coldham, A. J. Collis, R. J. Mould, R. E. Rathmell, J. Chem. Soc., Perkin Trans. 1 1995, 2739-2745.

${ }^{10}$ N. Yamagiwa, H. Qin, S. Matsunaga, M. Shibasaki, J. Am. Chem. Soc. 2005, 127, 13419-13427.
} 
tert-Butyl aziridine-2-carboxylate

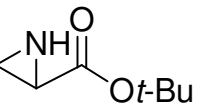

Table 1, entry 13

The general procedure for aziridination using $\mathrm{DppONH}_{2}, \mathrm{NMM}$ and $\mathrm{CH}_{2} \mathrm{Cl}_{2}$ was followed. The aziridine $(27.5 \mathrm{mg}, 0.192 \mathrm{mmol}, 80 \%)$ was isolated as a clear oil (care should be taken as the aziridine is volatile). IR (neat) $\vee 2980,1724,1457,1239,1158 \mathrm{~cm}^{-1}$. ${ }^{1} \mathrm{H} \mathrm{NMR}\left(400 \mathrm{MHz}, \mathrm{CDCl}_{3}\right)$ : $\delta 2.45(\mathrm{dd}, 1 \mathrm{H}, J=5.4,3.0 \mathrm{~Hz}, \mathrm{CHNH}), 1.94(\mathrm{dd}, 1 \mathrm{H}, J=3.0,1.5 \mathrm{~Hz}$, $\mathrm{CHNH}), 1.81(\mathrm{~d}, 1 \mathrm{H}, J=5.4 \mathrm{~Hz}, \mathrm{CHNH}), 1.50\left(\mathrm{~s}, 9 \mathrm{H}, \mathrm{C}\left(\mathrm{CH}_{3}\right)_{3}\right), 1.35(\mathrm{br}, 1 \mathrm{H}, \mathrm{NH}) .{ }^{13} \mathrm{C}$ NMR $\left(100 \mathrm{MHz}, \mathrm{CDCl}_{3}\right) \delta 172.1,82.2,29.9,28.1,27.0$. HRMS (CI, $\left.\mathrm{NH}_{3}\right): m / z\left[\mathrm{M}^{+}+\mathrm{H}\right]$ calcd for $\mathrm{C}_{7} \mathrm{H}_{14} \mathrm{NO}_{2}$ : 144.1025; found: 144.1027 .

\section{tert-Butyl 3-phenylaziridine-2-carboxylate}<smiles>CCCOC(=O)[C@@H]1NC1c1ccccc1</smiles>

Table 1, entry 14

The general procedure for aziridination using $\mathrm{DppONH}_{2}, \mathrm{NMM}$ and $\mathrm{CH}_{2} \mathrm{Cl}_{2}$ was followed. The aziridine $(8.5 \mathrm{mg}, 0.039 \mathrm{mmol}, 32 \%)$ was isolated as a clear oil. IR (neat) $\vee 3283,2980,2934,1721,1230,1157$ $\mathrm{cm}^{-1} .{ }^{1} \mathrm{H}$ NMR $\left(400 \mathrm{MHz}, \mathrm{CDCl}_{3}\right): \delta 7.34-7.27(\mathrm{~m}, 5 \mathrm{H}, \mathrm{PhH}), 3.17$ (br s, $1 \mathrm{H}, \mathrm{CHNH}), 2.50$ (br s, 1H, CHNH), $1.82(\mathrm{br}, 1 \mathrm{H}, \mathrm{NH}), 1.50\left(\mathrm{~s}, 9 \mathrm{H}, \mathrm{C}\left(\mathrm{CH}_{3}\right)_{3}\right) .{ }^{13} \mathrm{C} \mathrm{NMR}$ $\left(100 \mathrm{MHz}, \mathrm{CDCl}_{3}\right) \delta 170.9,138.3,128.6,127.8,126.3,82.5,40.6,40.1,28.2$. HRMS (CI, $\left.\mathrm{NH}_{3}\right): m / z\left[\mathrm{M}^{+}+\mathrm{H}\right]$ calcd for $\mathrm{C}_{13} \mathrm{H}_{18} \mathrm{NO}_{2}: 220.1338$; found: 220.1342 .

\section{Sub-stoichiometric amine in the aziridination of chalcones}

General procedure for the aziridination using $\mathrm{DppONH}_{2}$ and sub-stoichiometric amine. NMP $(3.1 \mu \mathrm{L}, 0.030 \mathrm{mmol}, 25 \mathrm{~mol} \%)$ was added to a solution of $\mathrm{DppONH}_{2}$ $\mathrm{NH} \| \quad(7.25 \mathrm{mg}, 0.0315 \mathrm{mmol})$ in DMF $(2 \mathrm{~mL})$ at $\mathrm{rt}$. The clear solution was allowed to stir for $30 \mathrm{~min}$, then $\mathrm{NaOH}(9.6 \mathrm{mg}, 0.24 \mathrm{mmol})$ and chalcone (25 $\mathrm{mg}, 0.12 \mathrm{mmol}$ ) were added sequentially. The reaction was allowed to stir at $\mathrm{rt}$ for $1 \mathrm{~h}$, then $\mathrm{DppONH}_{2}(7.25 \mathrm{mg}, 0.0315 \mathrm{mmol})$ was added. This was repeated a further twice, after which tlc indicated the reaction was nearly complete. The reaction mixture was quenched by the addition of saturated $\mathrm{NH}_{4} \mathrm{Cl}$ solution $(2 \mathrm{~mL})$. The solution was extracted with $\mathrm{CH}_{2} \mathrm{Cl}_{2}(3 \times 10 \mathrm{~mL})$, the organics combined, dried $\left(\mathrm{Na}_{2} \mathrm{SO}_{4}\right)$ and concentrated in vacuo. Purification by column chromatography $(100 \%$ petroleum ether to $10 \%$ EtOAc in petroleum ether) gave the aziridine $(17.6 \mathrm{mg}, 0.079 \mathrm{mmol}, 66 \%)$ as a white solid. Data matches previously reported values.

\section{In situ hydrazinium salt formation and enantioselective aziridination of chalcone}

$\mathrm{Ph}^{\prime \prime}<\mathrm{NH}_{\mathrm{Ph}} \begin{aligned} & \text { Quinine }(41 \mathrm{mg}, 0.13 \mathrm{mmol}) \text { was added in one portion to a solution of } \\ & \text { DppONH } \\ & \text { was allowed to stir for } 30 \mathrm{~min} \text {, then isopropanol }(29 \mu \mathrm{L}, 0.25 \mathrm{mmol}), \mathrm{NaH}\end{aligned}$ (60\% dispersion in mineral oil, $10 \mathrm{mg}, 0.25 \mathrm{mmol}$ ) and chalcone $(25 \mathrm{mg}, 0.12 \mathrm{mmol}$ ) were added sequentially. The mixture was allowed to stir at $\mathrm{rt}$ for $18 \mathrm{~h}$ (tlc indicated reaction was complete), then quenched by the addition of saturated $\mathrm{NH}_{4} \mathrm{Cl}$ solution $(2 \mathrm{~mL})$. The solution was extracted with $\mathrm{CH}_{2} \mathrm{Cl}_{2}(3 \times 10 \mathrm{~mL})$, the organics combined, dried $\left(\mathrm{Na}_{2} \mathrm{SO}_{4}\right)$ and concentrated in vacuo. Purification by column chromatography (100\% petroleum ether to $10 \%$ EtOAc in petroleum ether) gave the aziridine (17.2 $\mathrm{mg}, 0.08 \mathrm{mmol}, 64 \%)$ as a white solid. Data matches previously reported values. $[\alpha]_{\mathrm{D}}^{22}-139\left(c 0.66, \mathrm{CHCl}_{3}\right)$ for a sample of $56 \%$ ee (lit. $[\alpha]_{\mathrm{D}}^{22}+251.3\left(c 0.85, \mathrm{CHCl}_{3}\right)$ for a sample of $\left.95 \% \mathrm{ee}^{11}\right)$. 
Optical purity determined by chiral HPLC analysis in comparison with authentic racemic material.

Conditions: 93:7 hexane:IPA, $0.8 \mathrm{~mL} / \mathrm{min}, 254 \mathrm{~nm}, \mathrm{AD}-\mathrm{H}$.

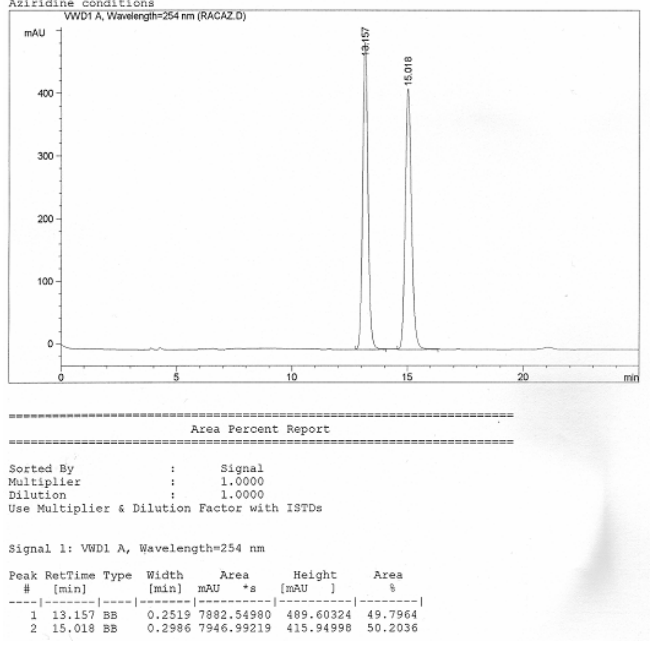

authentic racemic
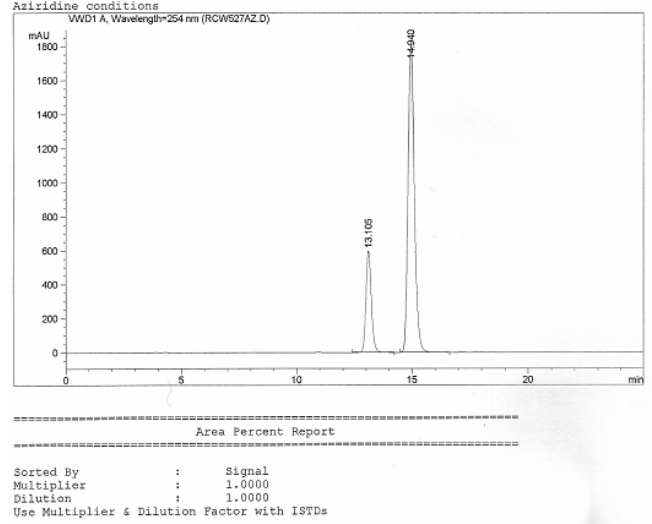

Signal 1: vwD1 A, wavelength=254 $\mathrm{nm}$

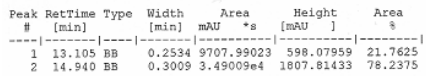

$56 \%$ ee

\section{Investigation into the enantioselective aziridination of chalcone using quinine}

Table 5. Enantioselective aziridination of chalcone using quinine ${ }^{a}$

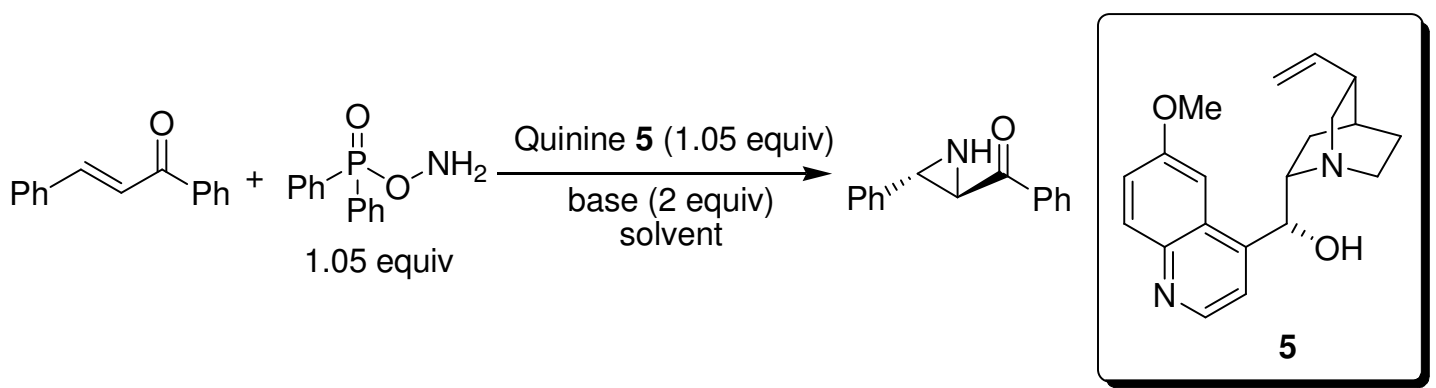

\begin{tabular}{ccccc}
\hline Entry & Solvent & Base & ${\text { Yield }(\%)^{b}}^{b}$ & ee $(\%)^{c}$ \\
\hline 1 & $\mathrm{CH}_{2} \mathrm{Cl}_{2}$ & $\mathrm{NaH} / i-\mathrm{PrOH}$ & 64 & 56 \\
2 & $\mathrm{CH}_{2} \mathrm{Cl}_{2}$ & $\mathrm{NaOH}$ & 59 & 46 \\
3 & Toluene & $\mathrm{NaH} / i-\mathrm{PrOH}$ & 64 & 41 \\
4 & Toluene & $\mathrm{NaOH}$ & 43 & 7 \\
5 & $\mathrm{THF}$ & $\mathrm{NaH} / i-\mathrm{PrOH}$ & 0 & - \\
6 & $\mathrm{THF}$ & $\mathrm{NaOH}$ & 0 & - \\
7 & $\mathrm{DMF}$ & $\mathrm{NaOH}$ & 36 & 0 \\
\hline
\end{tabular}

${ }^{a}$ Reactions performed on 0.12 mmol scale. ${ }^{b}$ Isolated yields after column chromatography. ${ }^{c}$ Determined by chiral HPLC, for conditions see above. 
A. Armstrong et al, Supporting Information, Page S10
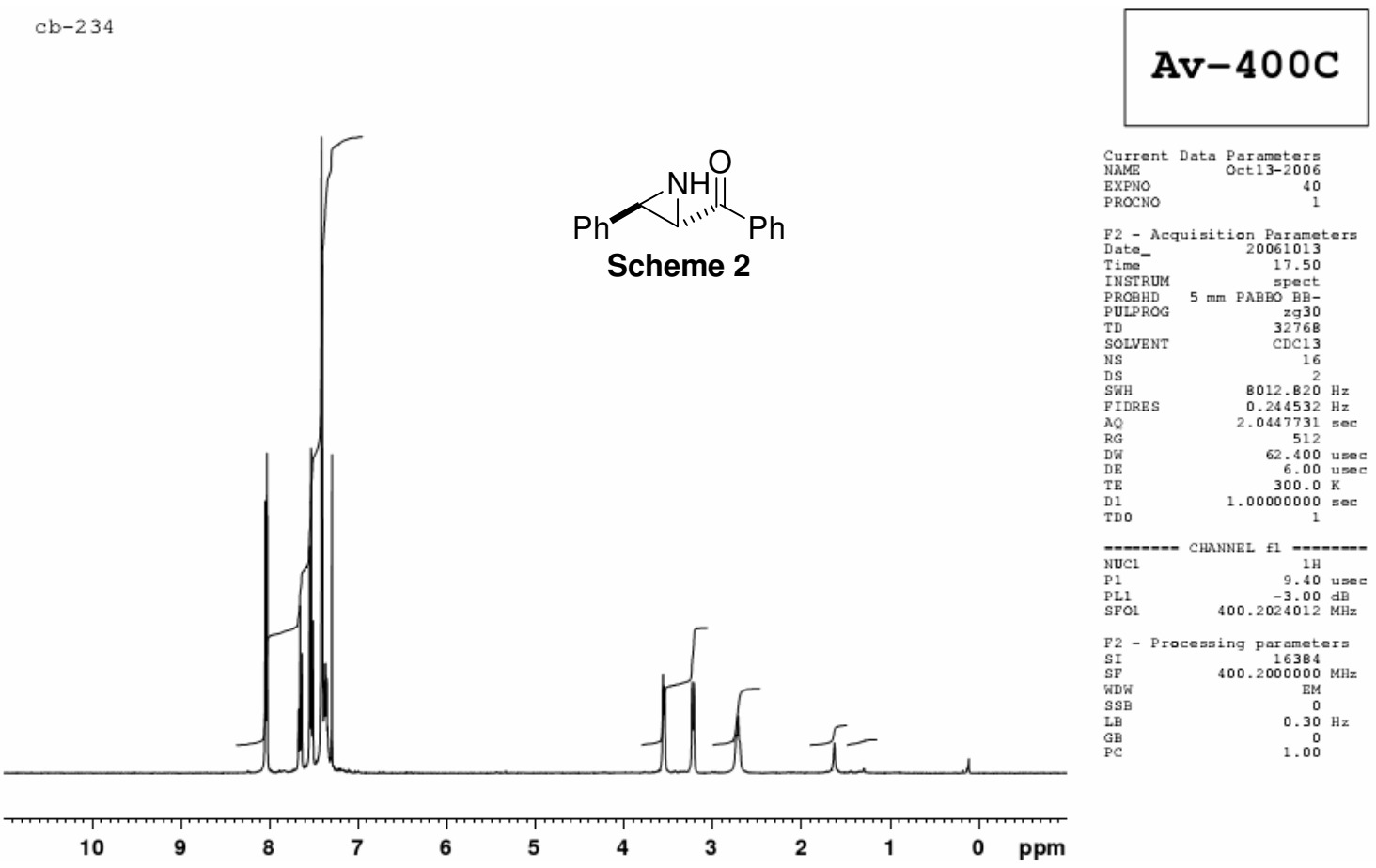

$\mathrm{cb}-148$

\section{Av-400A}
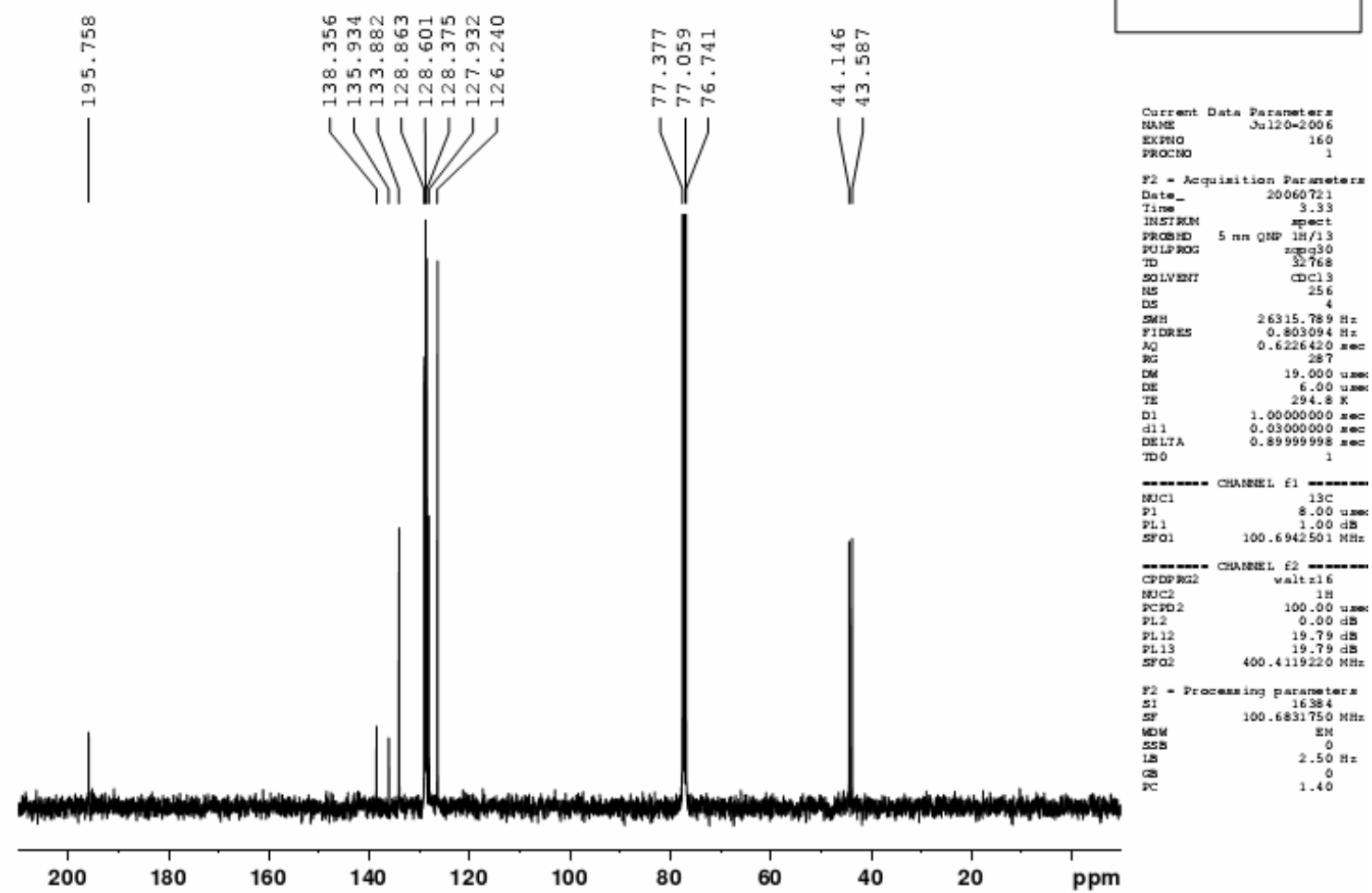
A. Armstrong et al, Supporting Information, Page S11

C Baxter CB-052 in CDC13; 1H spectrum using DRX400; Mar $17 / 170$

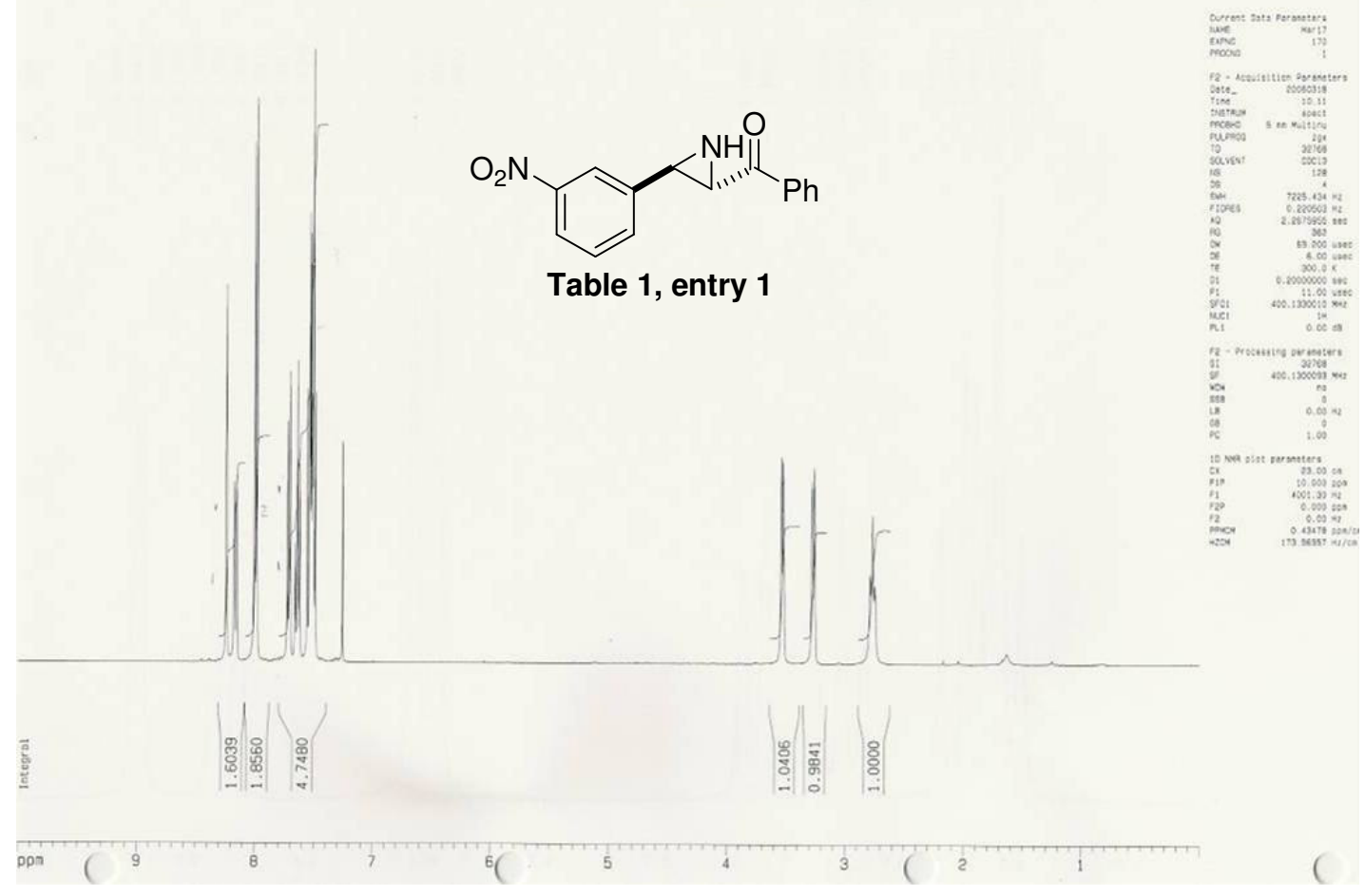

C Baxter $\mathrm{CB}-052$ in $\mathrm{CDCl3} ; 13 \mathrm{C}\{1 \mathrm{H}\}$ spectrum using DRX400; Mar 17/171

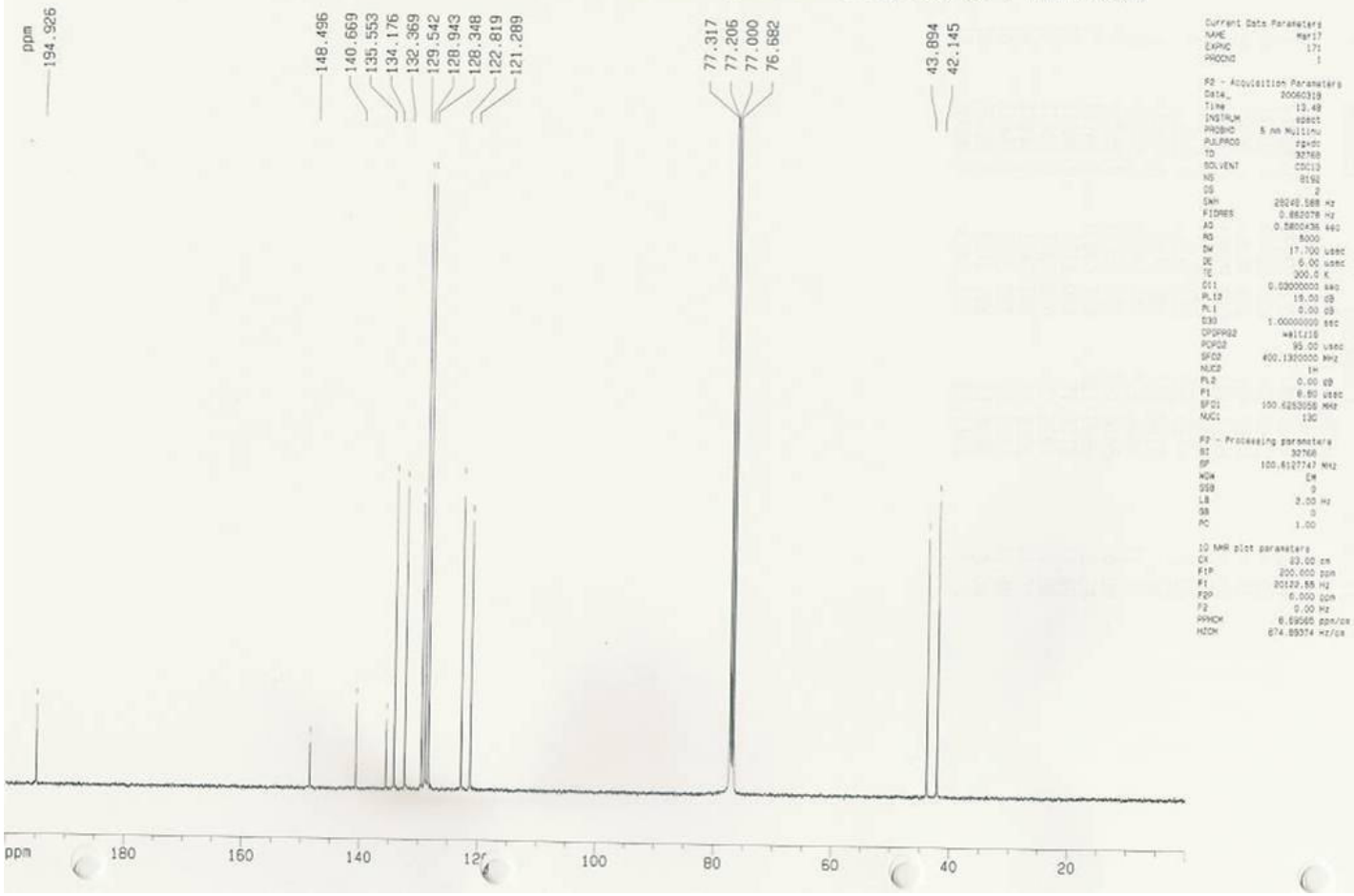


A. Armstrong et al, Supporting Information, Page S12
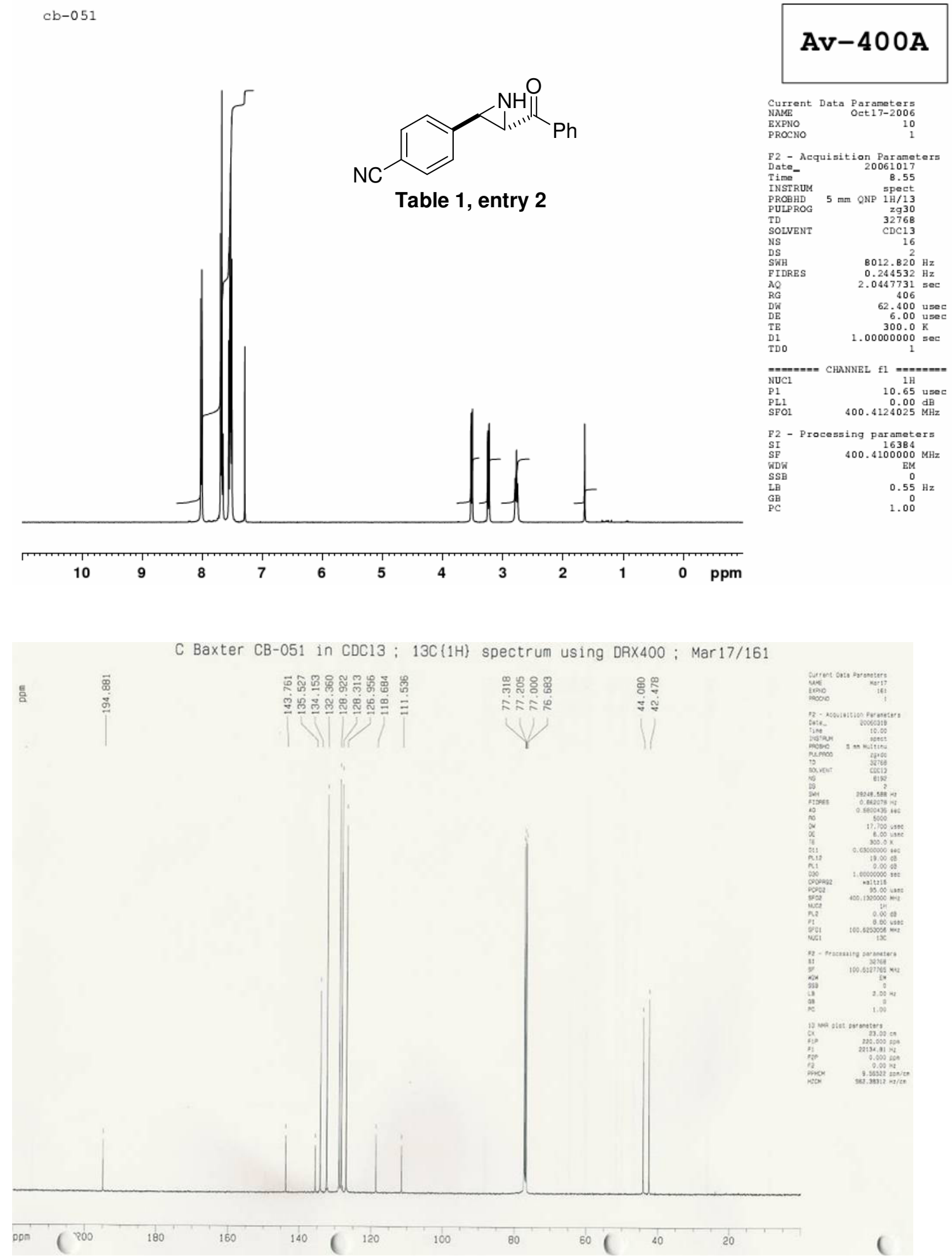
A. Armstrong et al, Supporting Information, Page S13
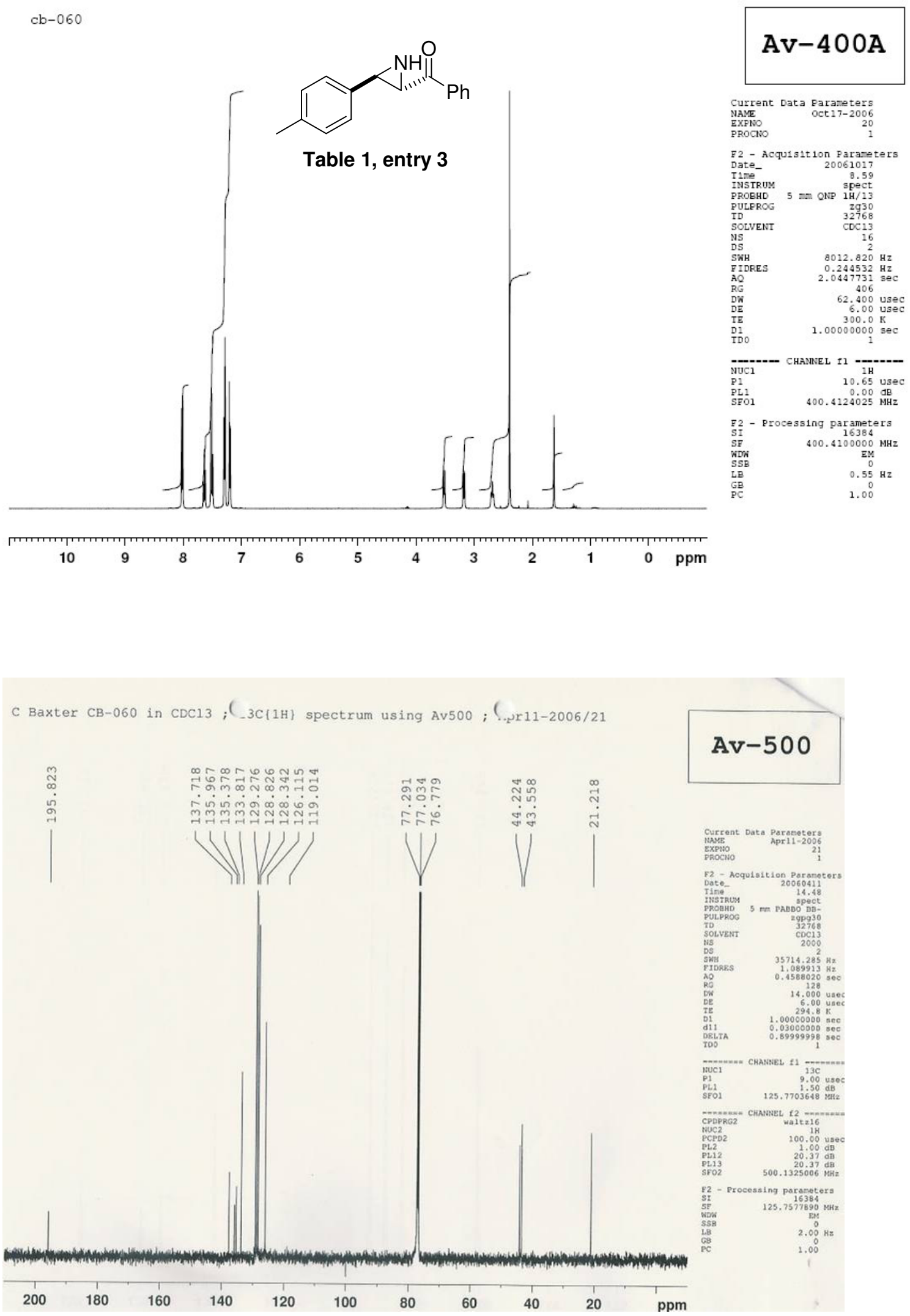
A. Armstrong et al, Supporting Information, Page S14
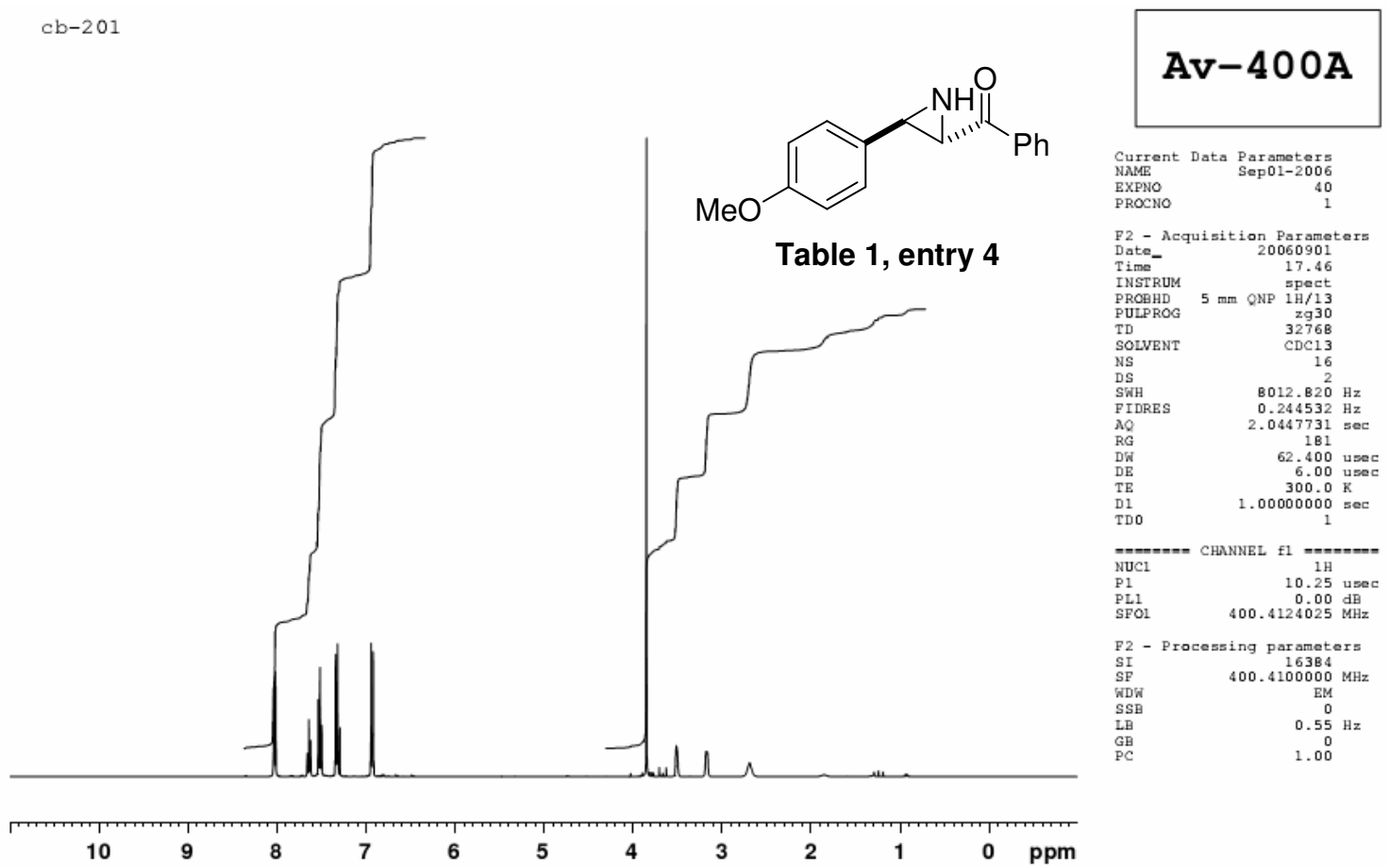

$c b-201$

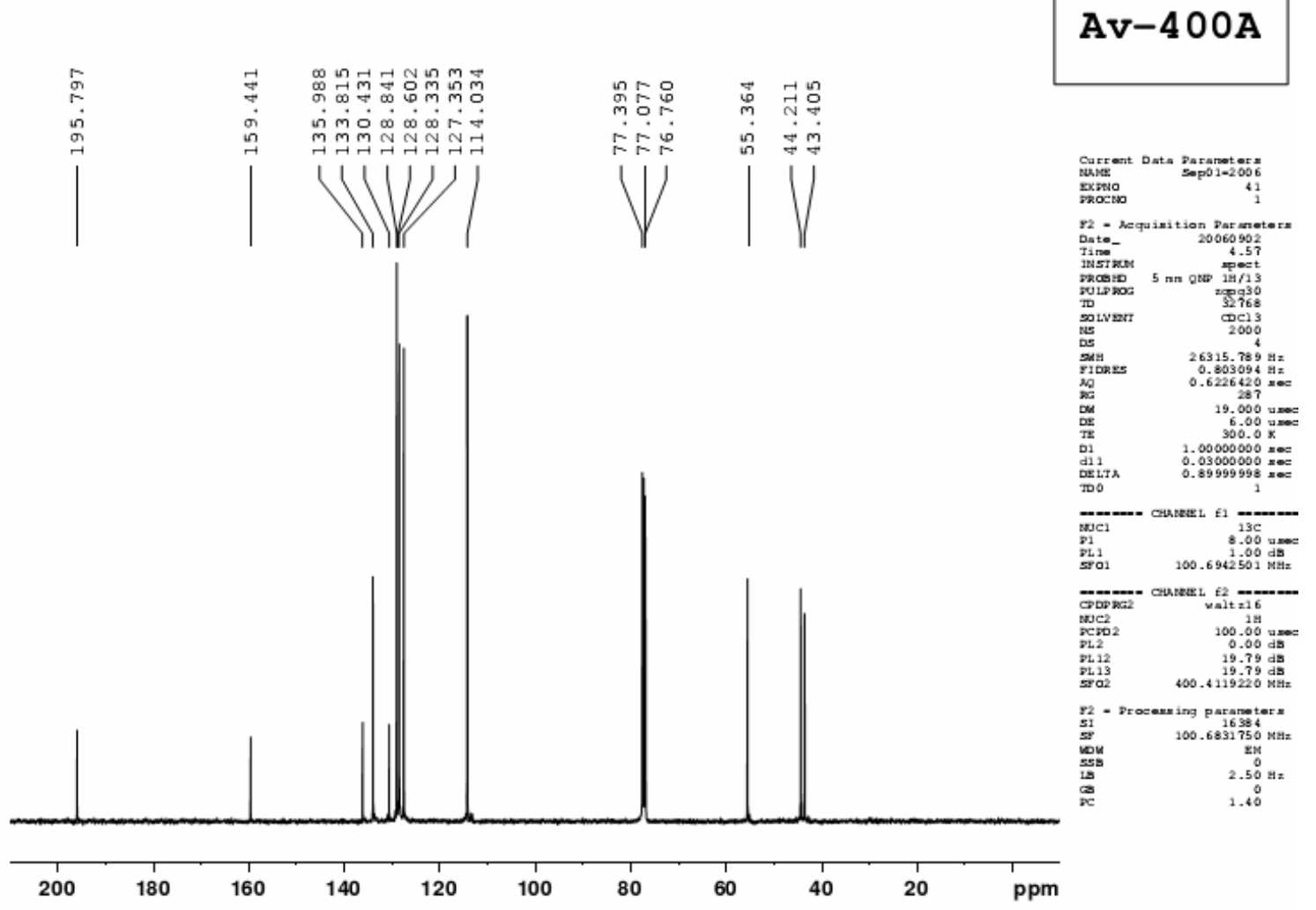


A. Armstrong et al, Supporting Information, Page S15

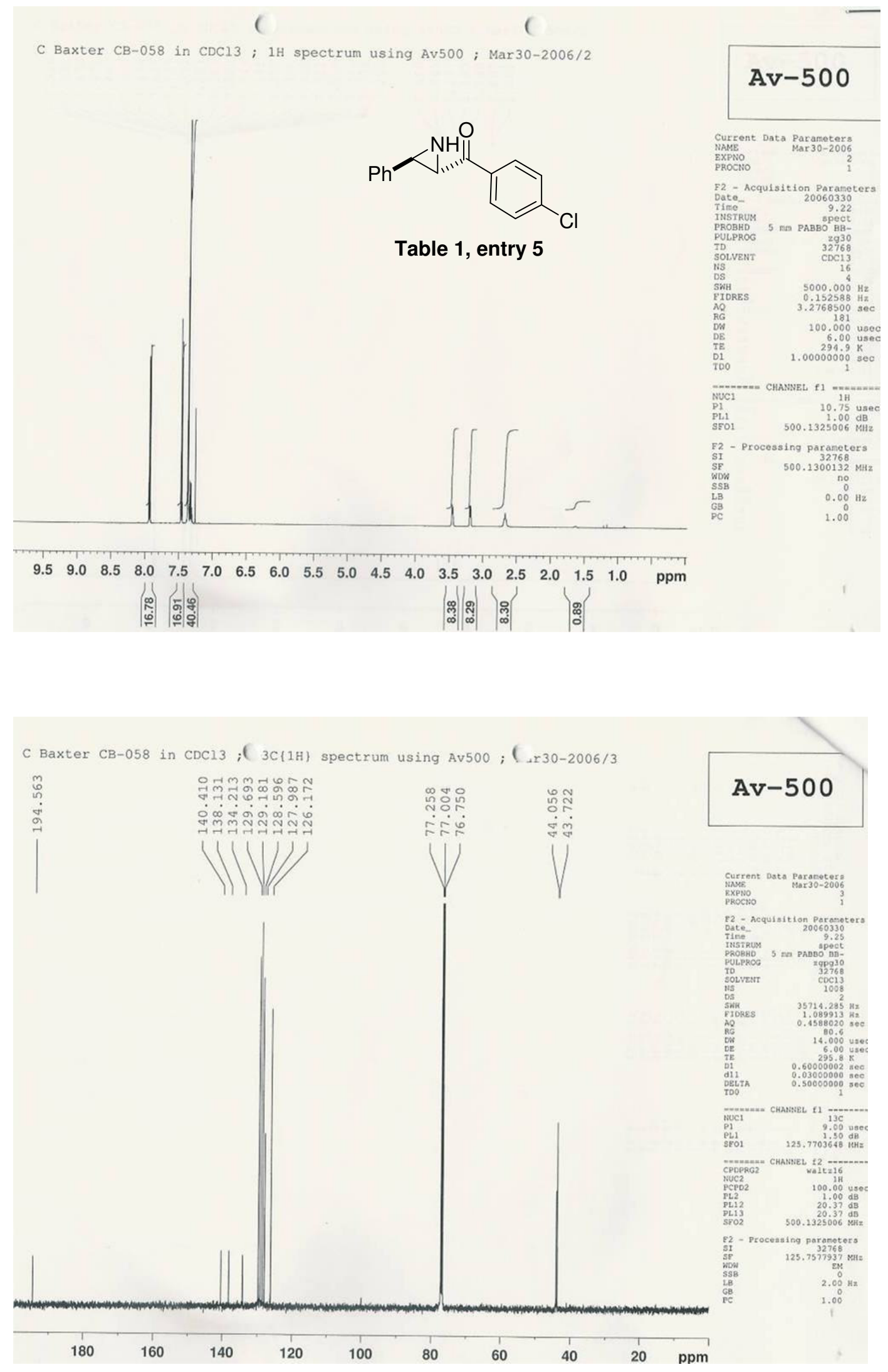


A. Armstrong et al, Supporting Information, Page S16

C Baxter $\mathrm{CB}-059$ in $\mathrm{CDC} 13$; $1 \mathrm{H}$ spectrum using Av500 ; Apr11-2006/10

$$
\mathrm{Av}-500
$$

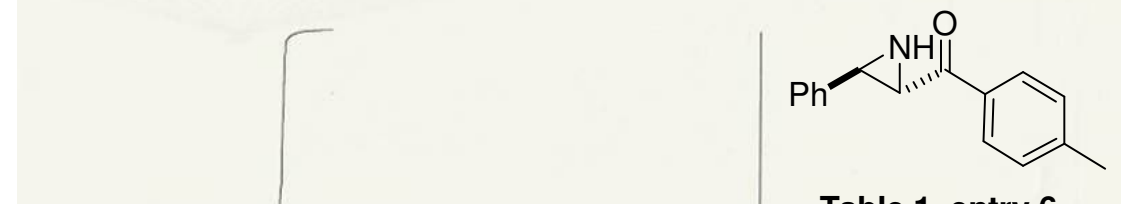

Table 1, entry 6

C Baxter $\mathrm{CB}-059$ in $\mathrm{CDCl} 3,\left({ }_{2} \mathrm{C}(1 \mathrm{H}\}\right.$ spectrum using Av500; .pr $11-2006 / 11$
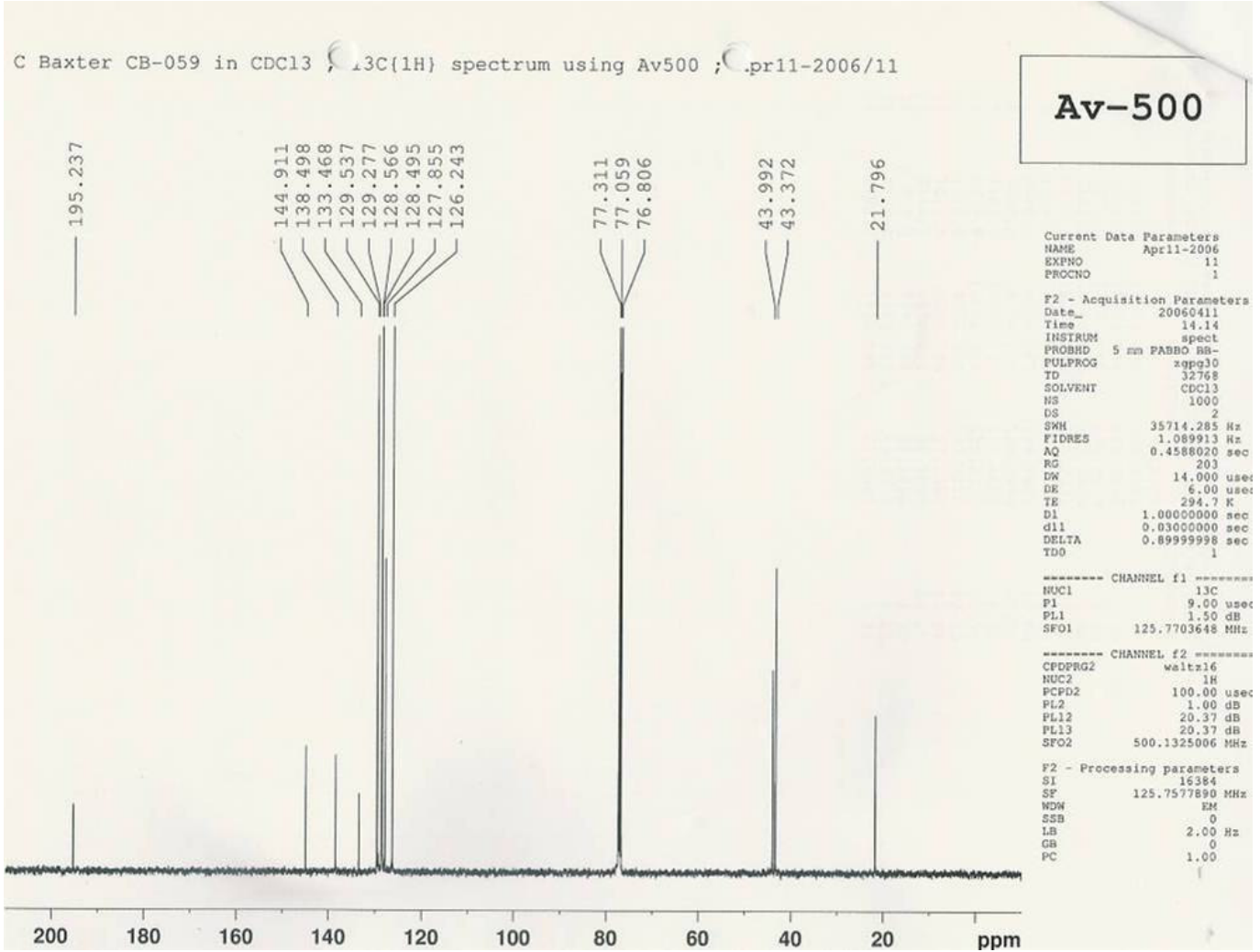


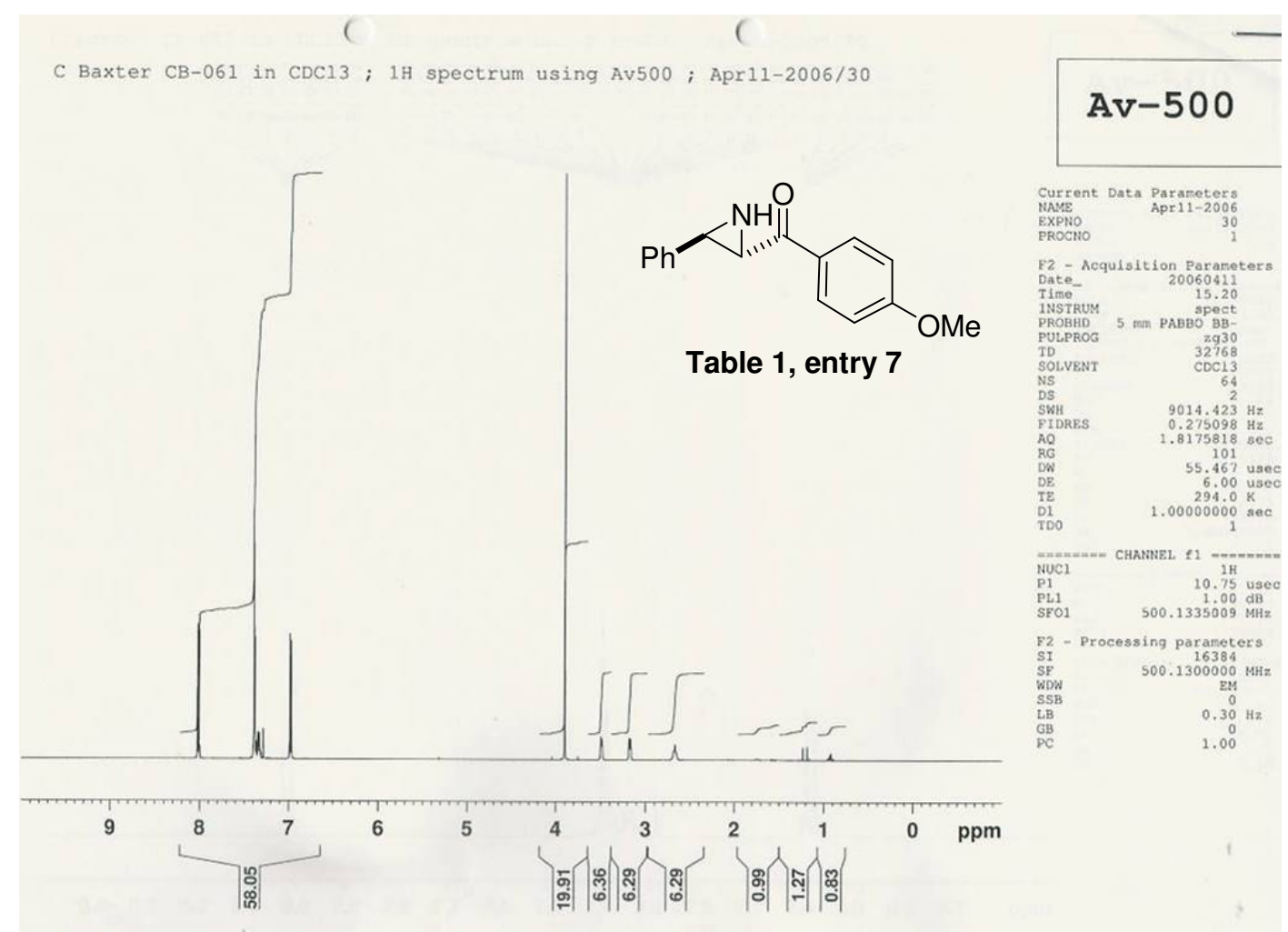

C Baxter $\mathrm{CB}-061$ in $\mathrm{CDC} 13 \mathrm{C}, 13 \mathrm{C}\{1 \mathrm{H}\}$ spectrum using Av500 , Apr11-2006/31
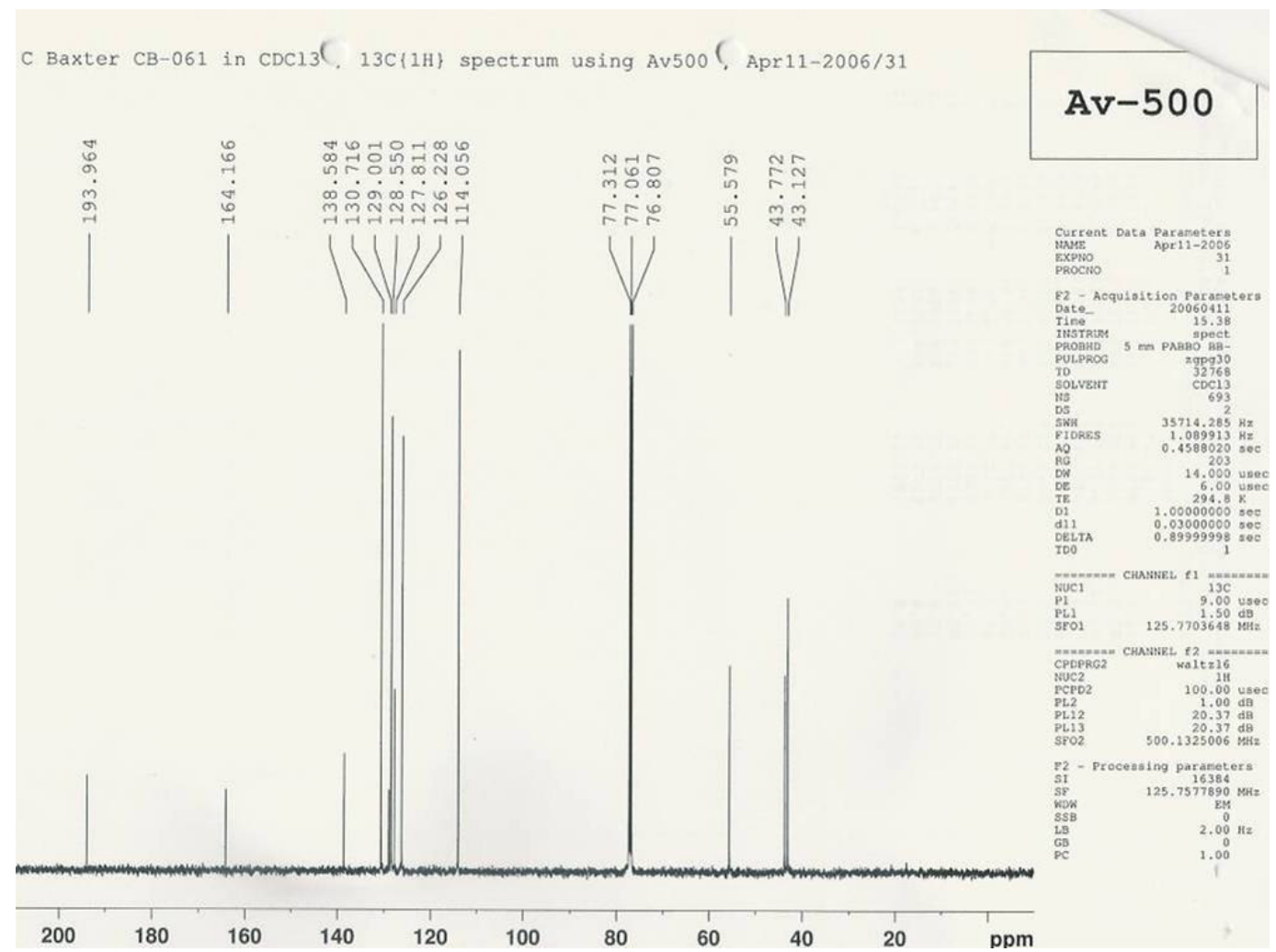
A. Armstrong et al, Supporting Information, Page S18
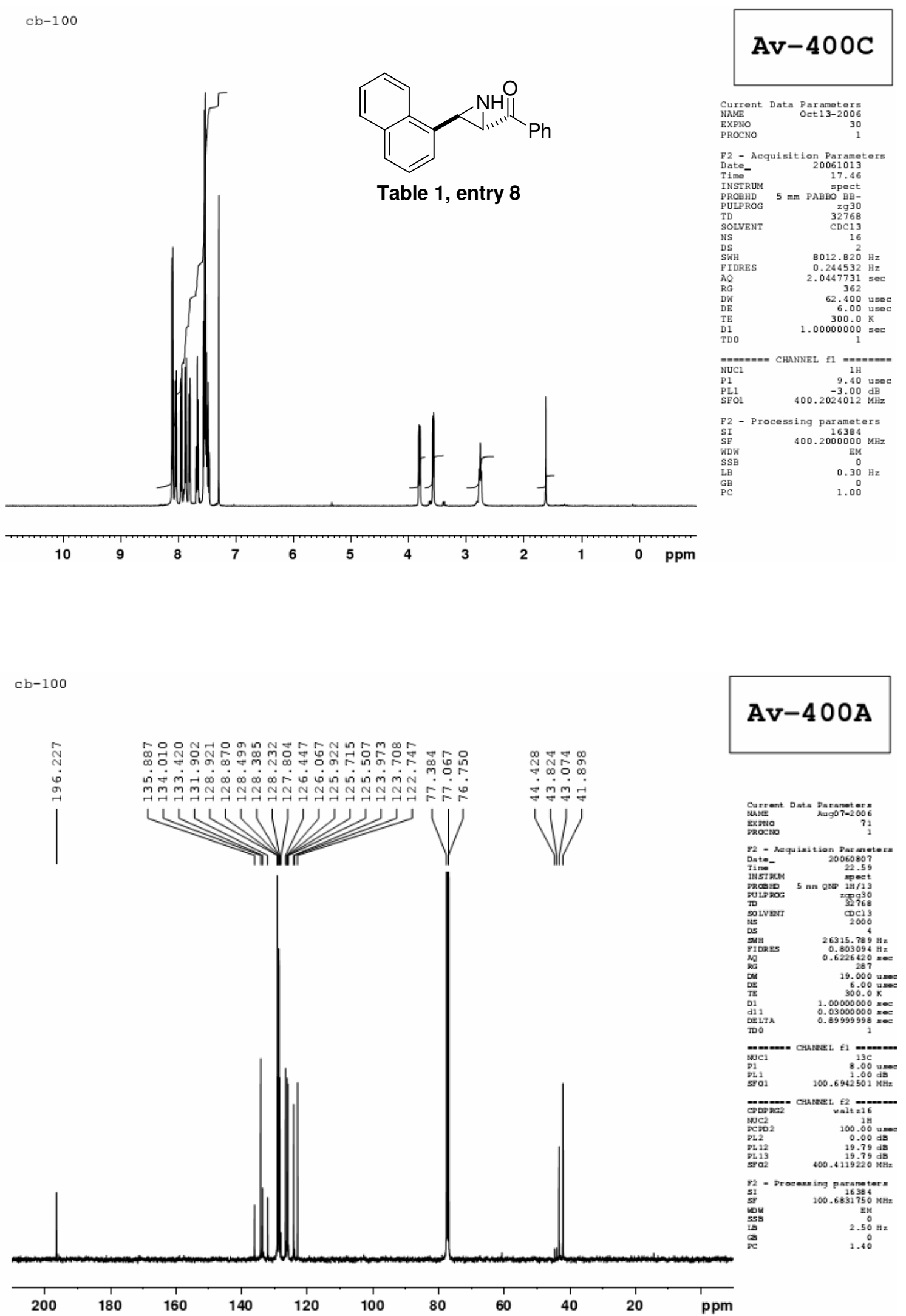
A. Armstrong et al, Supporting Information, Page S19
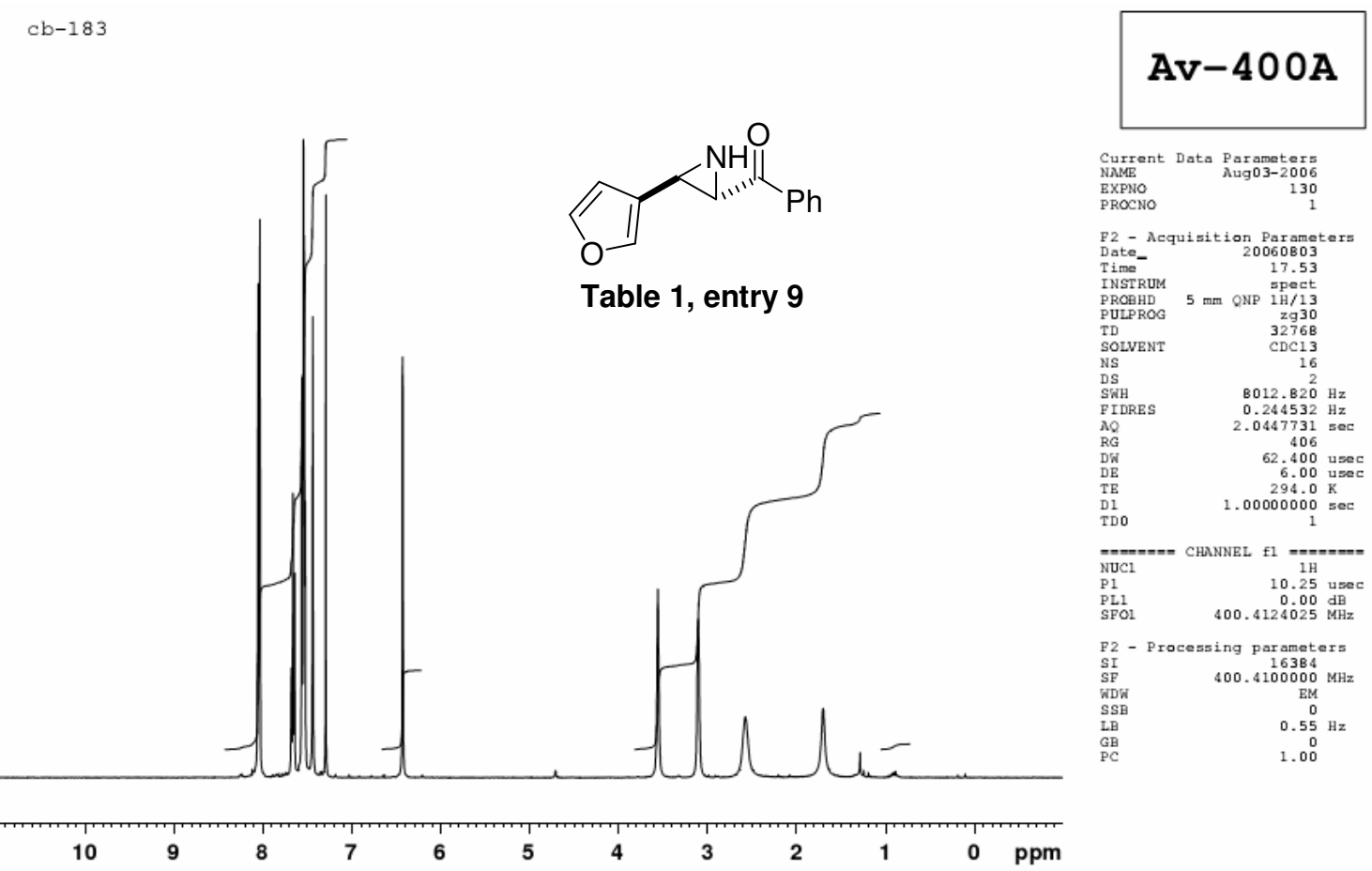

$\mathrm{cb}-183$

Av-400A
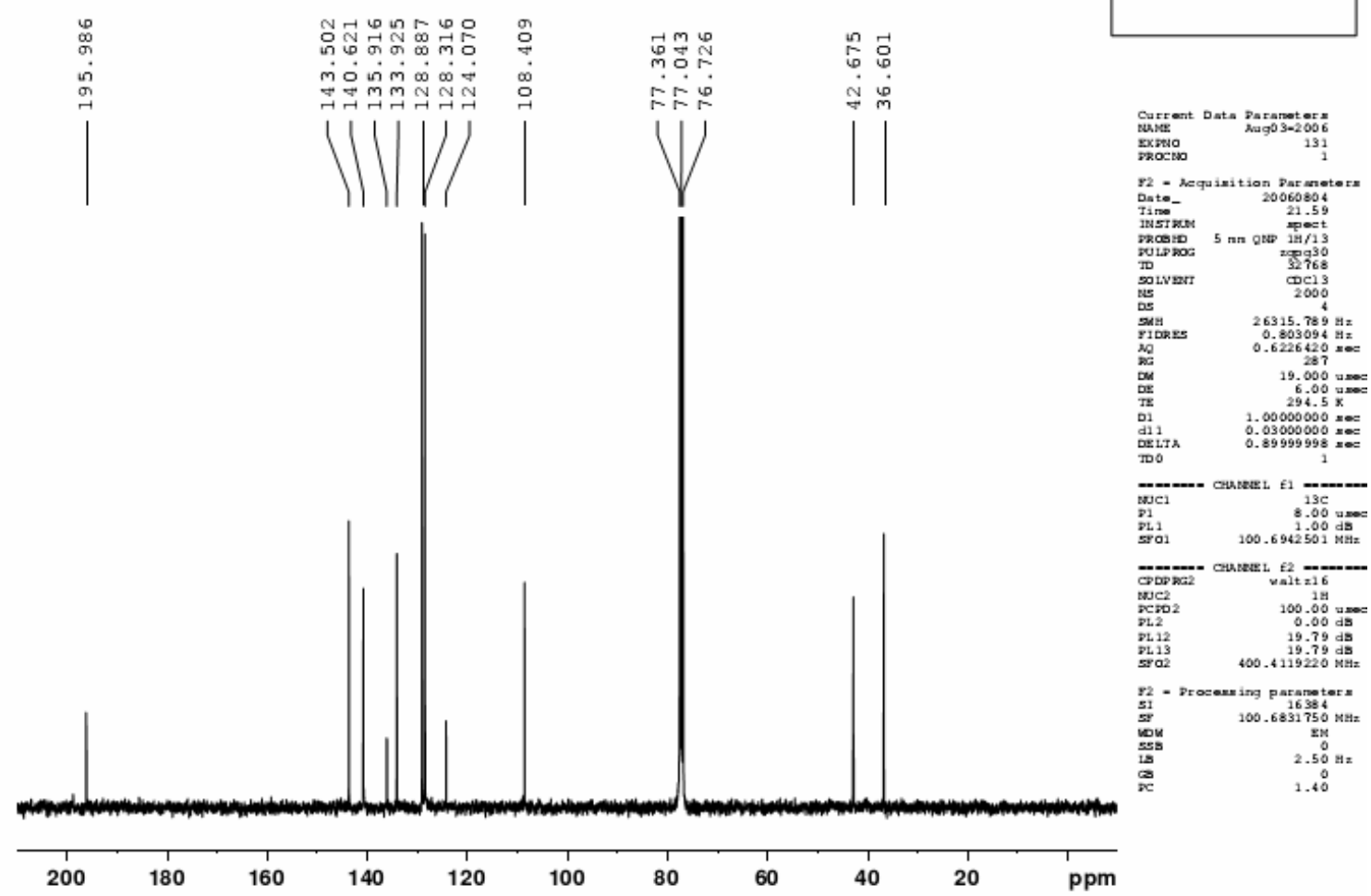
A. Armstrong et al, Supporting Information, Page S20

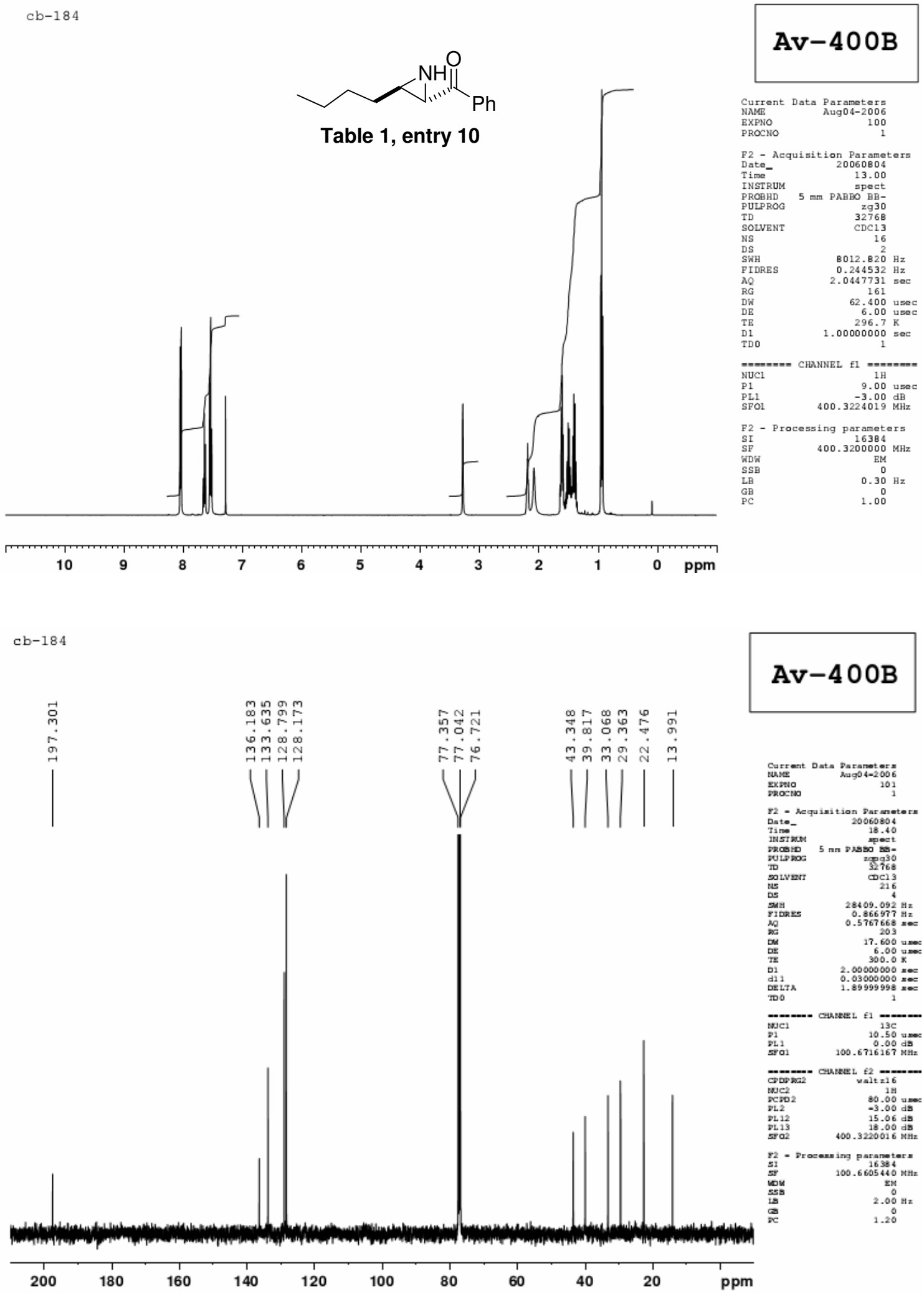


A. Armstrong et al, Supporting Information, Page S21

$c b-217$

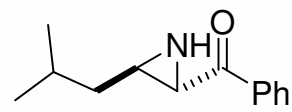

Table 1, entry 11

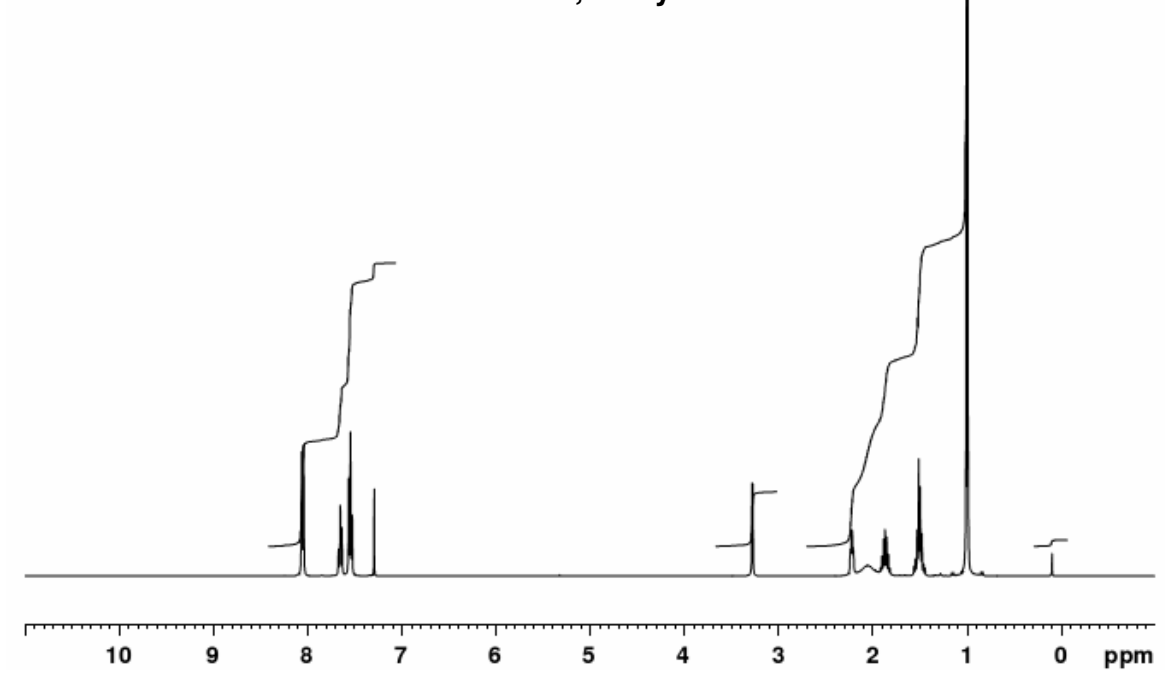

$\mathrm{cb}-217$

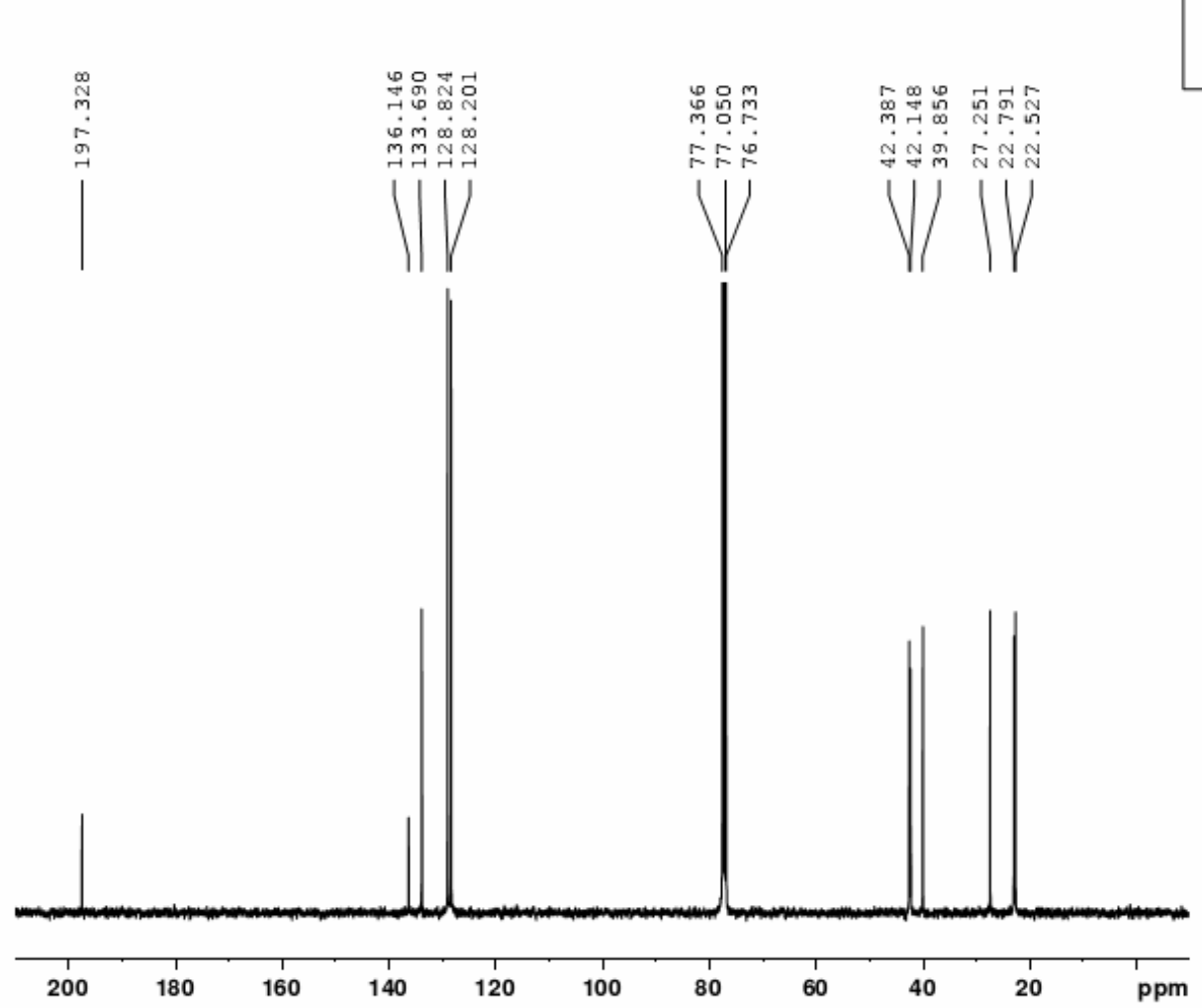

$\mathrm{Av}-400 \mathrm{~A}$

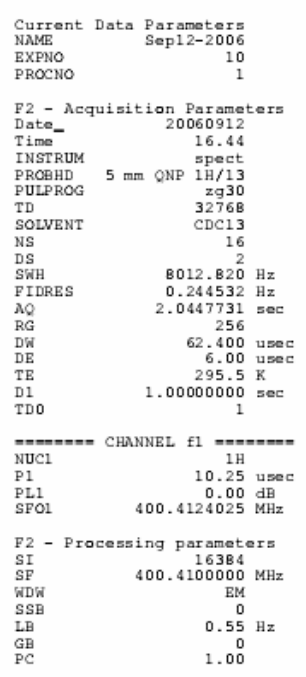

Av-400A

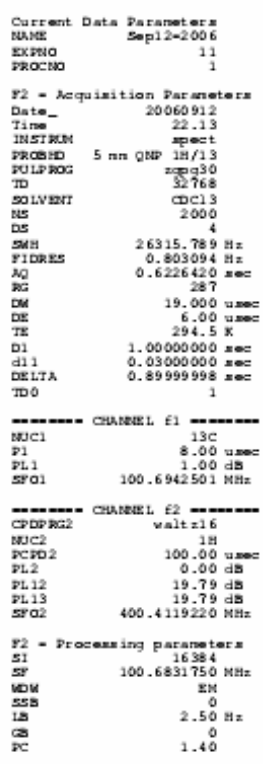


A. Armstrong et al, Supporting Information, Page S22
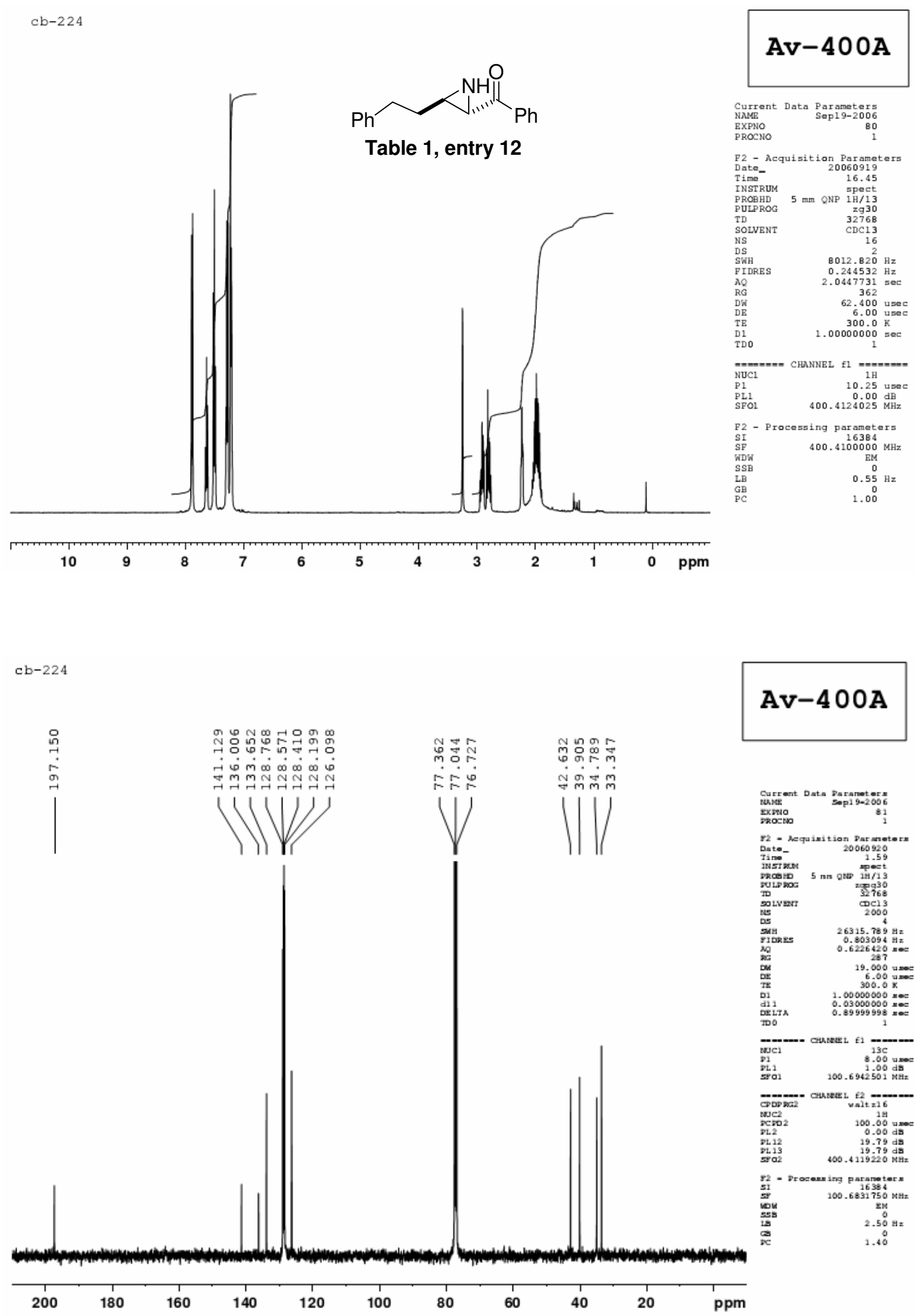
A. Armstrong et al, Supporting Information, Page S23
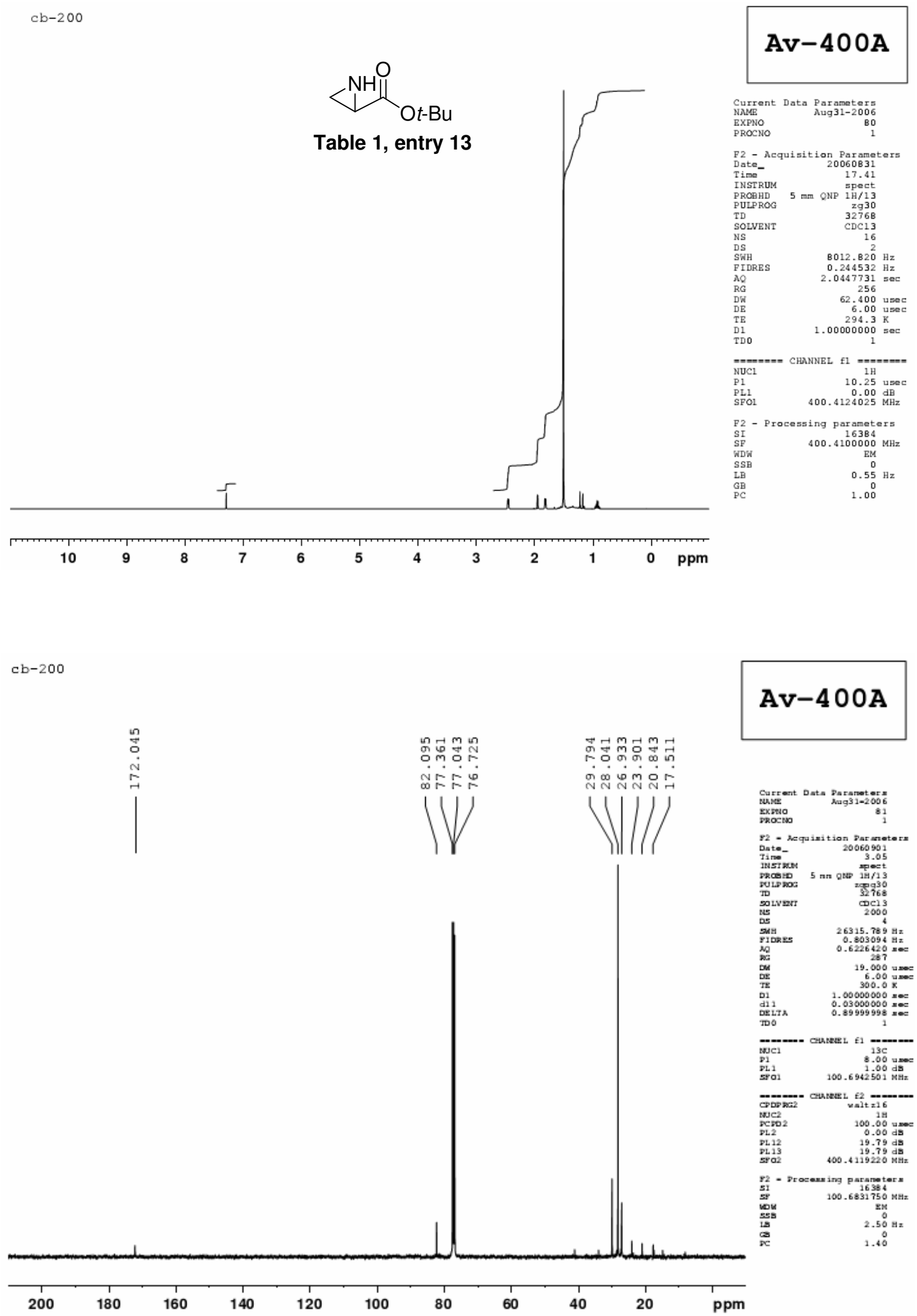
A. Armstrong et al, Supporting Information, Page S24
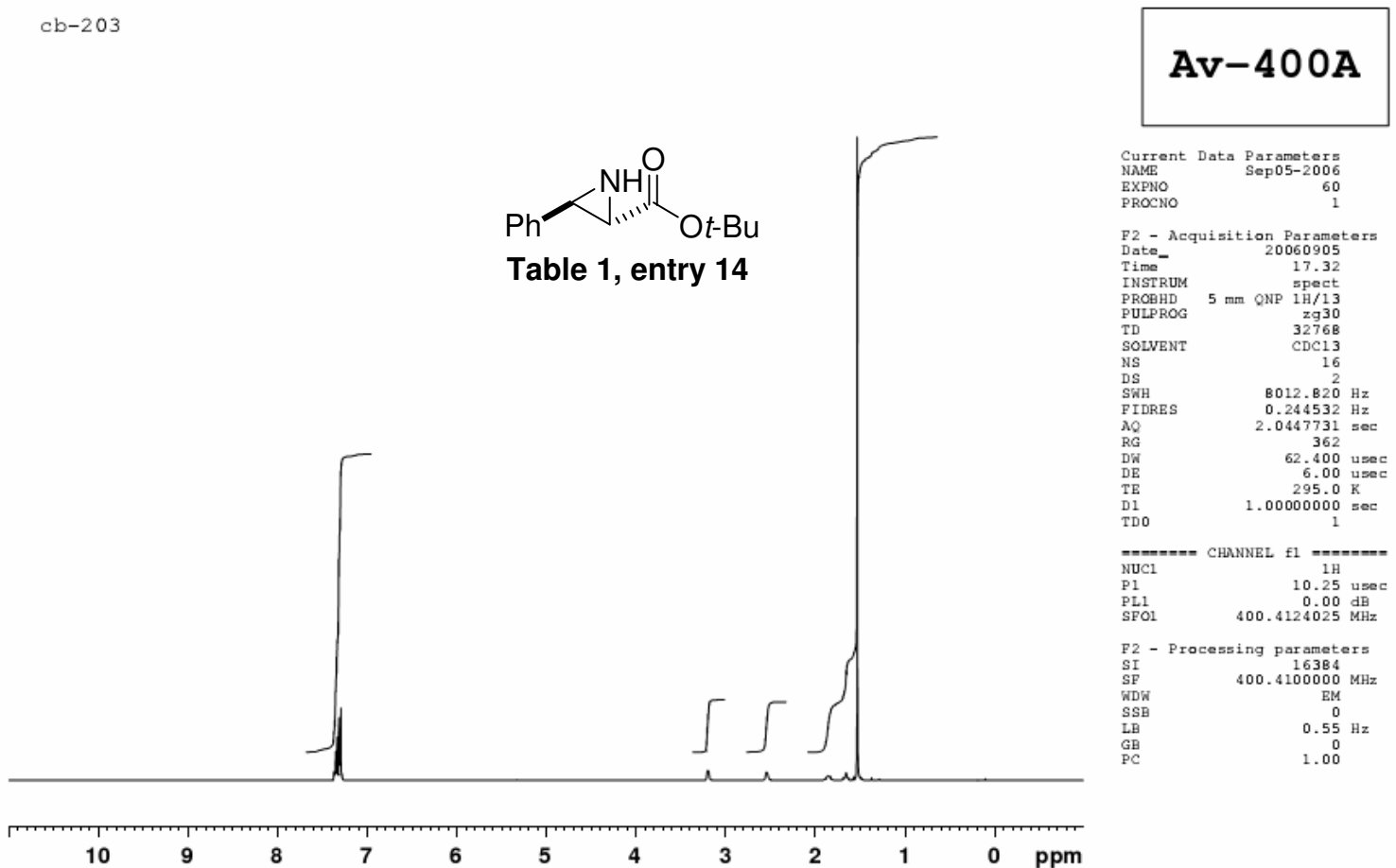

$c b-203$

Av-400A
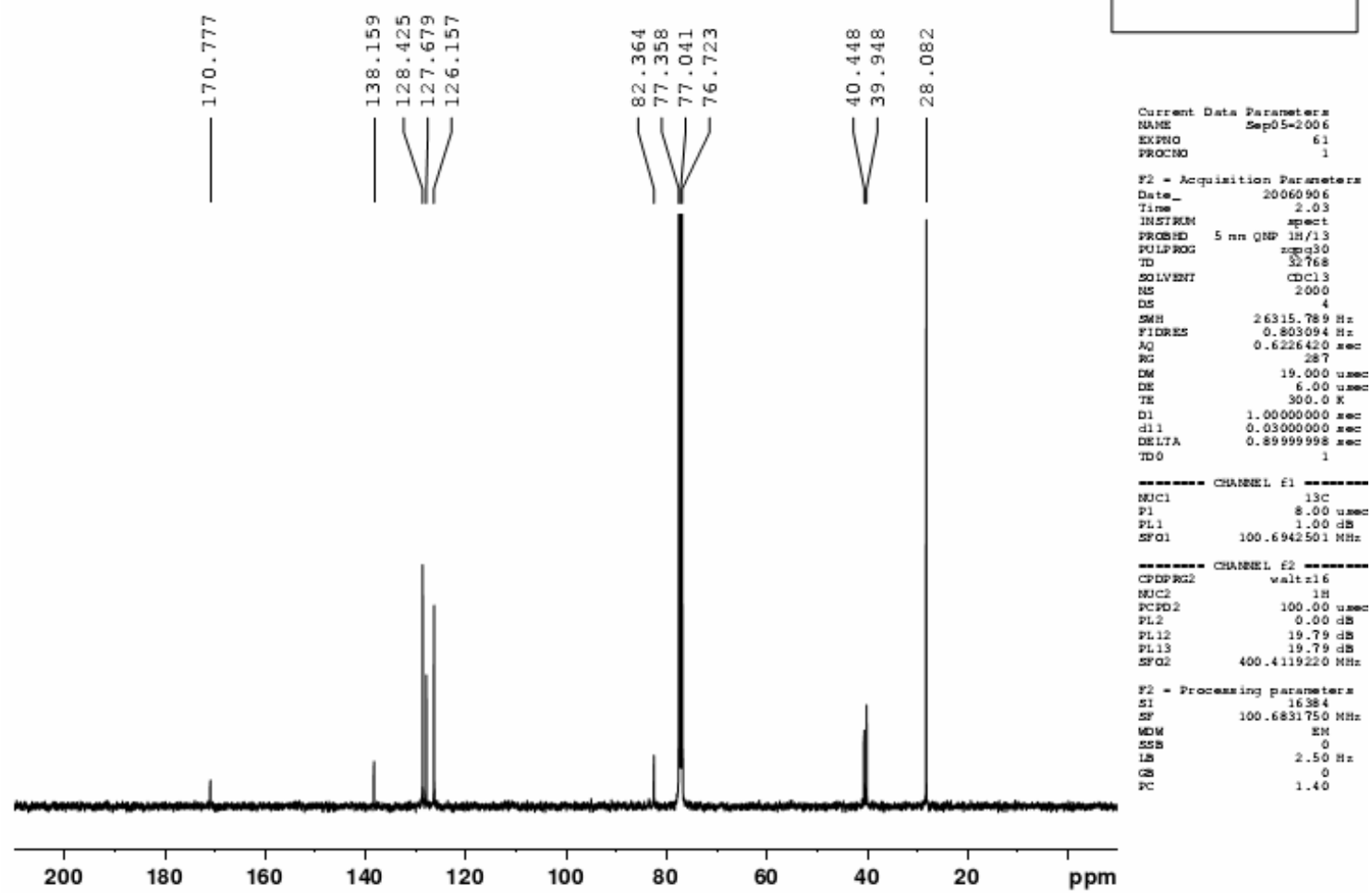